\title{
Two covering polynomials of a finite poset, with applications to root systems and ad-nilpotent ideals
}

\author{
DMitri Panyushev
}

\begin{abstract}
We introduce two polynomials (in $q$ ) associated with a finite poset $\mathcal{P}$ that encode some information on the covering relation in $\mathcal{P}$. If $\mathcal{P}$ is a distributive lattice, and hence $\mathcal{P}$ is isomorphic to the poset of dual order ideals in a poset $\mathcal{L}$, then these polynomials coincide and the coefficient of $q$ equals the number of $k$-element antichains in $\mathcal{L}$. In general, these two covering polynomials are different, and we introduce a deviation polynomial of $\mathcal{P}$, which measures the difference between these two. We then compute all these polynomials in the case, where $\mathcal{P}$ is one of the posets associated with an irreducible root system. These are 1) the posets of positive roots, 2) the poset of ad-nilpotent ideals, and 3) the poset of Abelian ideals.
\end{abstract}

AMS 2000 SUBJECT ClASSIFICATIONS: 06A07, 17B20, 20F55.

KEYWORDS AND PHRASES: Root system, ad-nilpotent ideal, graded poset, Hasse diagram.

\section{Introduction}

In this note, we associate two polynomials with a finite poset $\mathcal{P}$, study their properties, and determine these polynomials for some interesting posets related to root systems. Specifically, we mean the poset of positive roots, poset of $a d$-nilpotent ideals, and poset of abelian ideals, see definitions below.

Consider two statistics $\kappa$ and $\iota$ on $\mathcal{P}$. By definition, $\kappa(x), x \in \mathcal{P}$, is the number of elements of $\mathcal{P}$ that are covered by $x$, and $\iota(x)$ is the number of elements that cover $x$. The generating function associated with $\kappa$ (resp. $\iota$ ) is called the upper (resp. lower) covering polynomial of $\mathcal{P}$. That is, $\hat{\mathcal{K}}_{\mathcal{P}}(q)=$ $\sum_{x \in \mathcal{P}} q^{\kappa(x)}$ and $\check{\mathcal{K}}_{\mathcal{P}}(q)=\sum_{x \in \mathcal{P}} q^{\iota(x)}$. The upper covering polynomial, $\hat{\mathcal{K}}_{\mathcal{P}}$, has briefly been considered, without the adjective 'upper', in [16, Section 5]. It immediately follows from the definition that $\hat{\mathcal{K}}_{\mathcal{P}}(1)=\check{\mathcal{K}}_{\mathcal{P}}(1)$ and $\hat{\mathcal{K}}_{\mathcal{P}}^{\prime}(1)=$ $\check{\mathcal{K}}_{\mathcal{P}}^{\prime}(1)$. The last equality stems from the observation that both values equal the number of edges in the Hasse diagram of $\mathcal{P}$. Consequently, $\hat{\mathcal{K}}_{\mathcal{P}}(q)-$ $\check{\mathcal{K}}_{\mathcal{P}}(q)=(q-1)^{2} \mathcal{D}_{\mathcal{P}}(q)$ for some polynomial $\mathcal{D}_{\mathcal{P}}$, which is called the deviation polynomial of $\mathcal{P}$. 
In Section 2, we begin with some simple observations on these polynomials and then prove that $\hat{\mathcal{K}}_{\mathcal{P}} \equiv \check{\mathcal{K}}_{\mathcal{P}}$ if $\mathcal{P}$ is a distributive lattice (see Theorem 2.2). For a distributive lattice $\mathcal{P}$, the common covering polynomial is denoted by $\mathcal{K}_{\mathcal{P}}$.

Let $\Delta$ be an irreducible root system, $\Delta^{+}$a subset of positive roots, and $\Pi \subset \Delta^{+}$the set of simple roots. If $\Delta$ is reduced, then $\mathfrak{g}$ is the corresponding simple Lie algebra, with fixed Borel subalgebra $\mathfrak{b}$ corresponding to $\Delta^{+}$. We determine $\hat{\mathcal{K}}_{\mathcal{P}}, \check{\mathcal{K}}_{\mathcal{P}}$, and $\mathcal{D}_{\mathcal{P}}$ in the following cases:

1) $\mathcal{P}=\Delta^{+}$, equipped with the standard root order, see Section 3 ;

2) $\mathcal{P}$ is $J^{*}\left(\Delta^{+}\right)$or $\mathcal{P}=J^{*}\left(\Delta^{+} \backslash \Pi\right)$, where $J^{*}(\mathcal{L})$ stands for the poset of dual order ideals in $\mathcal{L}$. In the Lie algebra case, $J^{*}\left(\Delta^{+}\right)$is isomorphic to the poset of ad-nilpotent ideals of $\mathfrak{b}$, i.e., $\mathfrak{b}$-ideals in $\mathfrak{u}=[\mathfrak{b}, \mathfrak{b}]$, the nilradical of $\mathfrak{b}$; and $J^{*}\left(\Delta^{+} \backslash \Pi\right)$ is isomorphic to the poset of $\mathfrak{b}$-ideals in $[\mathfrak{u}, \mathfrak{u}]$. These two posets are also denoted by $\mathfrak{A} \mathfrak{d}$ and $\mathfrak{A d}_{0}$, respectively;

3) $\mathcal{P}=\mathfrak{A} \mathfrak{b}$, the subposet of $\mathfrak{A} \mathfrak{d}$ that consists of the abelian ideals of $\mathfrak{b}$. (An abelian ideal of $\mathfrak{b}$ is a subspace $\mathfrak{c} \subset \mathfrak{b}$ such that $[\mathfrak{b}, \mathfrak{c}] \subset \mathfrak{c}$ and $[\mathfrak{c}, \mathfrak{c}]=0$.) Whenever we wish to stress that these (po)sets depend on $\mathfrak{g}$, we write $\Delta(\mathfrak{g})$, $\mathfrak{A} \mathfrak{d}(\mathfrak{g})$, etc.

Let us briefly describe our results. For $\Delta^{+}$, we prove that $\operatorname{deg} \hat{\mathcal{K}}_{\Delta^{+}}=$ $\operatorname{deg} \check{\mathcal{K}}_{\Delta^{+}} \leqslant 3$ and the coefficients of $q^{3}$ in $\hat{\mathcal{K}}_{\Delta^{+}}$and $\check{\mathcal{K}}_{\Delta^{+}}$are equal. This implies that the deviation polynomial is a constant; namely, $\mathcal{D}_{\Delta^{+}}(q) \equiv \mathrm{rk} \Delta-$ 1. This also includes the only non-reduced irreducible root system $\mathbf{B} \mathbf{C}_{n}$. We show that the posets $\Delta^{+}\left(\mathbf{B C}_{n}\right), \Delta^{+}\left(\mathbf{B}_{n+1}\right) \backslash \Pi$, and $\Delta^{+}\left(\mathbf{C}_{n+1}\right) \backslash \Pi$ are isomorphic, which allows to reduce many problems on $\mathbf{B C}_{n}$ to $\mathbf{B}_{n+1}$ or $\mathbf{C}_{n+1}$.

Since $\mathfrak{A} \mathfrak{d}$ and $\mathfrak{A d}_{0}$ are distributive lattice, we have only two different covering polynomials for them. The polynomial $\mathcal{K}_{\mathfrak{A d}}$ appeared earlier under various guises in different theories in $[1,2,15,19]$. The coefficients of $\mathcal{K}_{\mathfrak{A d}}$ are the generalised Narayana numbers. A posteriori, it is known that $\hat{\mathcal{K}}_{\mathfrak{A d}}$ is palindromic, but no general explanation is available in the context of adnilpotent ideals. Explicit formulae for $\mathcal{K}_{\mathfrak{A d}_{0}}$ show that this polynomial is not always palindromic. (Our computation of $\mathcal{K}_{\mathfrak{A d}_{0}\left(\mathbf{D}_{n}\right)}$ relies on the conjectural relationship between the coefficients of $\mathcal{K}_{\mathfrak{A d}_{0}}(q)$ and the $\mathcal{F}$-triangle introduced by F. Chapoton [7], see Section 4.) However, the ratio $\hat{\mathcal{K}}^{\prime}(1) / \hat{\mathcal{K}}(1)$ is determined by similar rules in both cases. We notice that

$$
\frac{\mathcal{K}_{\mathfrak{A d}}^{\prime}(1)}{\mathcal{K}_{\mathfrak{A d}}(1)}=\frac{\#\left(\Delta^{+}\right)}{h} \quad \text { and } \quad \frac{\mathcal{K}_{\mathfrak{A d}_{0}}^{\prime}(1)}{\mathcal{K}_{\mathfrak{A d}_{0}}(1)}=\frac{\#\left(\Delta^{+} \backslash \Pi\right)}{h-1}
$$

where $h$ is the Coxeter number of $\Delta$. The first equality stems from the fact that $\mathcal{K}_{\mathfrak{A d}}$ is palindromic, of degree $n=\mathrm{rk} \Delta$ (although the fact that $\mathcal{K}_{\mathfrak{A d}}$ is 
palindromic is not explained yet). The reason for the validity of the second one is totally unclear.

The most interesting case is that of abelian ideals. Here the upper and lower covering polynomials are usually different. The reason is that although $\mathfrak{A} \mathfrak{b}$ is a meet semilattice, it is a distributive lattice if and only if $\Delta$ is of type $\mathbf{C}_{n}$ or $\mathbf{G}_{2}$. We develop some general theory for computing covering polynomials, which is based on a bijection between the abelian ideals and the minuscule elements of the affine Weyl group of $\Delta$. Let $I \subset \Delta^{+}$be an abelian ideal. Using the minuscule element corresponding to $I$, we define the shift vector $\mathbf{k}_{I}=\left(k_{0}, k_{1}, \ldots, k_{n}\right)$, where $k_{i} \in\{-1,0,1,2\}$, and prove that $\kappa(I)=\#\left\{i \mid k_{i}=-1\right\}$ and $\iota(I)=\#\left\{j \mid k_{j}=1\right\}$. We then describe a recursive procedure for computing all $\mathbf{k}_{I}$ starting from $I=\varnothing$. The procedure basically asserts that if $k_{i}=1$, then $\mathbf{k}_{I}$ can be replaced with $\mathbf{k}_{I}-\{$ the $i$-th column of the extended Cartan matrix of $\Delta\}$, see Section 5 for details.

We also present a method of calculation of $\check{\mathcal{K}}_{\mathfrak{A} \mathfrak{b}}$, which exploits the canonical mapping of $\mathfrak{A} \mathfrak{b} \backslash\{\varnothing\}$ onto the set of long positive roots [14]. For explicit computations with exceptional root systems, we use the general equalities $\hat{\mathcal{K}}_{\mathfrak{A b}}(1)=2^{n}[4,11]$ and $\hat{\mathcal{K}}_{\mathfrak{A} \mathfrak{b}}^{\prime}(1)=(n+1) 2^{n-2}[16]$; our calculations in the classical cases exploit standard matrix presentations of these Lie algebras and counting certain Ferrers diagrams.

Our computations show that, for many natural posets, the coefficients of $\mathcal{D}_{\mathcal{P}}$ are of the same sign. This includes $\mathfrak{A} \mathfrak{b}, \Delta^{+}, \Delta^{+} \cup\{0\}, \Delta^{+} \backslash \Pi$. It is likely that there could exist some general conditions on $\mathcal{P}$ guaranteeing that $\mathcal{D}_{\mathcal{P}}$ has the coefficients of the same sign. In Section 7 , we propose a condition of such sort.

\section{Definition and basic properties}

Let $(\mathcal{P}, \preccurlyeq)$ be a finite poset. Write $\mathcal{H}(\mathcal{P})$ for the Hasse diagram of $\mathcal{P}$ and $\mathcal{E}(\mathcal{P})$ for the set of edges of $\mathcal{H}(\mathcal{P})$. We regard $\mathcal{H}(\mathcal{P})$ as a digraph; if $x$ covers $y(x, y \in \mathcal{P})$, then the edge $(x, y)$ is depicted as $y \rightarrow x$ and we say that $(x, y)$ originates in $y$ and terminates in $x$.

For any $x \in \mathcal{P}$, let $\kappa(x)$ be the number of $y \in \mathcal{P}$ such that $y$ is covered by $x$, and $\iota(x)$ the number of $y \in \mathcal{P}$ such that $y$ covers $x$. In other words, $\kappa(x)$ (resp. $\iota(x)$ ) is the number of edges in $\mathcal{E}(\mathcal{P})$ that terminates (resp. originates) in $x$. We define two polynomials that encode some properties of the covering relation in $\mathcal{P}$. 


\section{Definition 1.}

(i) The upper covering polynomial of $\mathcal{P}$ is $\hat{\mathcal{K}}_{\mathcal{P}}(q)=\sum_{x \in \mathcal{P}} q^{\kappa(x)}$;

(ii) The lower covering polynomial of $\mathcal{P}$ is $\check{\mathcal{K}}_{\mathcal{P}}(q)=\sum_{x \in \mathcal{P}} q^{\iota(x)}$;

It follows that $\hat{\mathcal{K}}_{\mathcal{P}}(0)$ (resp. $\check{\mathcal{K}}_{\mathcal{P}}(0)$ ) is the number of the minimal (resp. maximal) elements of $\mathcal{P}$. In general, these polynomials are different; they may even have different degrees. However, one readily deduces from the definition that

$$
\left.\hat{\mathcal{K}}_{\mathcal{P}}(q)\right|_{q=1}=\left.\check{\mathcal{K}}_{\mathcal{P}}(q)\right|_{q=1}=\# \mathcal{P} \text { and }\left.\frac{d}{d q} \hat{\mathcal{K}}_{\mathcal{P}}(q)\right|_{q=1}=\left.\frac{d}{d q} \check{\mathcal{K}}_{\mathcal{P}}(q)\right|_{q=1}=\# \mathcal{E}(\mathcal{P}) .
$$

Hence $\hat{\mathcal{K}}_{\mathcal{P}}(q)-\check{\mathcal{K}}_{\mathcal{P}}(q)=(q-1)^{2} \mathcal{D}_{\mathcal{P}}(q)$ for some polynomial $\mathcal{D}_{\mathcal{P}}$. We will say that $\mathcal{D}_{\mathcal{P}}$ is the deviation polynomial of $\mathcal{P}$. The following is straightforward.

Lemma 2.1. We have the following properties

(i) If $\mathcal{P}=\mathcal{P}_{1}+\mathcal{P}_{2}$, then $\hat{\mathcal{K}}_{\mathcal{P}}=\hat{\mathcal{K}}_{\mathcal{P}_{1}}+\hat{\mathcal{K}}_{\mathcal{P}_{2}}$, and likewise for $\check{\mathcal{K}}$ and $\mathcal{D}$;

(ii) If $\mathcal{P}=\mathcal{P}_{1} \times \mathcal{P}_{2}$, then $\hat{\mathcal{K}}_{\mathcal{P}}=\hat{\mathcal{K}}_{\mathcal{P}_{1}} \hat{\mathcal{K}}_{\mathcal{P}_{2}}$, $\check{\mathcal{K}}_{\mathcal{P}}=\check{\mathcal{K}}_{\mathcal{P}_{1}} \check{\mathcal{K}}_{\mathcal{P}_{2}}$, and $\mathcal{D}_{\mathcal{P}}=$ $\hat{\mathcal{K}}_{\mathcal{P}_{1}} \mathcal{D}_{\mathcal{P}_{2}}+\check{\mathcal{K}}_{\mathcal{P}_{2}} \mathcal{D}_{\mathcal{P}_{1}}=\check{\mathcal{K}}_{\mathcal{P}_{1}} \mathcal{D}_{\mathcal{P}_{2}}+\hat{\mathcal{K}}_{\mathcal{P}_{2}} \mathcal{D}_{\mathcal{P}_{1}}$.

We are going to investigate how properties of $\mathcal{P}$ are reflected in $\hat{\mathcal{K}}_{\mathcal{P}}, \check{\mathcal{K}}_{\mathcal{P}}, \mathcal{D}_{\mathcal{P}}$.

Theorem 2.2. Let $\mathcal{P}$ be a distributive lattice. Then $\hat{\mathcal{K}}_{\mathcal{P}}=\check{\mathcal{K}}_{\mathcal{P}}$. More precisely, if $\mathcal{P} \simeq J(\mathcal{L})$, then the coefficient of $q^{k}$ equals the number of $k$-element antichains in $\mathcal{L}$.

Proof. By Birkhoff's theorem for finite distributive lattices, $\mathcal{P}$ is isomorphic to the poset of order ideals of a unique poset $\mathcal{L}$, i.e., $\mathcal{P} \simeq J(\mathcal{L})$, see e.g. [22, Theorem 3.4.1]. If $I$ is an order ideal of $\mathcal{L}$, then the set of maximal elements of $I, \max (I)$, is an antichain of $\mathcal{L}$. And the same is true for the set of minimal elements of $\mathcal{L} \backslash I, \min (\mathcal{L} \backslash I)$. It easily follows from Definiton 1 that regarding $I$ as an element of $\mathcal{P}$ we have $\iota(I)=\# \max (I)$ and $\kappa(I)=$ $\# \min (\mathcal{L} \backslash I)$. Conversely, each antichain in $\mathcal{L}$ occurs as both $\max (I)$ and $\min (\mathcal{L} \backslash J)$ for suitable order ideals $I, J$. This means that both covering polynomials essentially count all the antichains of $\mathcal{L}$ with respect to their cardinality.

Remark. More generally, the equality $\hat{\mathcal{K}}_{\mathcal{P}}=\check{\mathcal{K}}_{\mathcal{P}}$ holds if $\mathcal{P}$ is a modular lattice [8]. This result of Dilworth is also discussed in [22, Ex. 3.38.5].

Below, it will be more convenient for us to think of a distributive lattice as the poset of dual order (= upper) ideals. The distributive lattice of upper 
ideals of a poset $\mathcal{L}$ is denoted by $J^{*}(\mathcal{L})$. Then $\mathcal{L}$ is being restored as the set of meet-irreducibles in $J^{*}(\mathcal{L})$. If $I \in J^{*}(\mathcal{L})$, then $\kappa(I)=\# \min (I)$ and $\iota(I)=$ $\# \max (\mathcal{L} \backslash I)$. Note also that the posets $J^{*}(\mathcal{L})$ and $J\left(\mathcal{L}^{o p}\right)$ are canonically isomorphic.

The following is clear:

Proposition 2.3. If $\mathcal{P}$ admits an order-reversing bijection, then $\hat{\mathcal{K}}_{\mathcal{P}}=\check{\mathcal{K}}_{\mathcal{P}}$.

Example 2.4. $1^{o}$. Let $(W, S)$ be a finite Coxeter group. Consider $W$ as poset under the Bruhat-Chevalley ordering ' $\leqslant$ '. It is easily seen that $W$ is not a lattice. But the mapping $w \mapsto w w_{0}$, where $w_{0} \in W$ is the longest element, yields an order-reversing bijection of $(W, \leqslant)$. Hence $\hat{\mathcal{K}}_{W}(q)=\check{\mathcal{K}}_{W}(q)$. More generally, such an equality also holds for the poset $W / W_{J}$, where $J \subset S$ and $W_{J}$ is the corresponding parabolic subgroup of $W$.

$2^{o}$. Let $\mathcal{P}$ be an arbitrary poset and $\mathcal{P}^{o p}$ the opposite poset. Then $\hat{\mathcal{K}}_{\mathcal{P}}=$ $\check{\mathcal{K}}_{\mathcal{P}^{o p}}$ and $\check{\mathcal{K}}_{\mathcal{P}}=\hat{\mathcal{K}}_{\mathcal{P}_{\text {op }}}$. Hence $\mathcal{D}_{\mathcal{P}}=-\mathcal{D}_{\mathcal{P}^{o p}}$. It then follows from Lemma 2.1 that $\mathcal{D}_{\mathcal{P} \times \mathcal{P}^{o p}}=0$. One may also notice that $\mathcal{P} \times \mathcal{P}^{o p}$ admits an orderreversing involution.

Example 2.5. As we have shown, if $\mathcal{P}=J^{*}(\mathcal{L})$, then $\mathcal{D}_{\mathcal{P}}=0$. Let $\hat{0}$ and $\hat{1}$ denote the maximal and minimal element of $\mathcal{P}$, respectively. Set $\mathcal{P}^{\prime}=\mathcal{P} \backslash\{\hat{0}\}$ and $\mathcal{P}^{\prime \prime}=\mathcal{P} \backslash\{\hat{1}\}$. It is easy to realise the effect of these procedures for both covering polynomials.

- Let $m$ be the number of maximal elements of $\mathcal{L}$. Then $\hat{\mathcal{K}}_{\mathcal{P}^{\prime}}(q)=$ $\mathcal{K}_{\mathcal{P}}(q)-m q+m-1$ and $\check{\mathcal{K}}_{\mathcal{P}^{\prime}}(q)=\mathcal{K}_{\mathcal{P}}(q)-q^{m}$. Hence $\hat{\mathcal{K}}_{\mathcal{P}^{\prime}}(q)-\check{\mathcal{K}}_{\mathcal{P}^{\prime}}(q)=$ $q^{m}-m q+(m-1)$ and $\mathcal{D}_{\mathcal{P}^{\prime}}(q)=q^{m-2}+2 q^{m-3}+\cdots+(m-2) q+(m-1)$. In particular, $\mathcal{D}_{\mathcal{P}^{\prime}} \equiv 0$ if and only if $m=1$. This is not surprising, because if $\mathcal{L}$ has a unique maximal element, say $a_{1}$, then $\mathcal{P} \backslash\{\hat{0}\} \simeq J^{*}\left(\mathcal{L} \backslash\left\{a_{1}\right\}\right)$.

- Since $J^{*}(\mathcal{L}) \backslash\{\hat{1}\} \simeq J\left(\mathcal{L}^{o p}\right) \backslash\{\hat{0}\}$, the formulae for $\mathcal{P}^{\prime \prime}$ are similar. Only the roles of $\hat{\mathcal{K}}$ and $\check{\mathcal{K}}$ are reversed, and in place of $m$ we need the number, say $l$, of minimal elements of $\mathcal{L}$. Therefore, $\mathcal{D}_{\mathcal{P}^{\prime \prime}}(q)=$ $-\left(q^{l-2}+2 q^{l-3}+\cdots+(l-2) q+(l-1)\right)$.

From the definition of $\mathcal{D}_{\mathcal{P}}$, it follows that $\mathcal{D}_{\mathcal{P}}(1)=\frac{1}{2}\left(\hat{\mathcal{K}}_{\mathcal{P}}^{\prime \prime}(1)-\check{\mathcal{K}}_{\mathcal{P}}^{\prime \prime}(1)\right)$. Therefore

$$
\begin{aligned}
\mathcal{D}_{\mathcal{P}}(1)= & \frac{\#\left\{\left(x, y_{1}, y_{2}\right) \in \mathcal{P}^{3} \mid y_{1} \rightarrow x, y_{2} \rightarrow x\right\}}{2} \\
& -\frac{\#\left\{\left(x_{1}, x_{2}, y\right) \in \mathcal{P}^{3} \mid y \rightarrow x_{1}, y \rightarrow x_{2}\right\}}{2},
\end{aligned}
$$


where it is assumed that $y_{1} \neq y_{2}$ and $x_{1} \neq x_{2}$. In other words,

$$
2 \mathcal{D}_{\mathcal{P}}(1)=\#\left\{0, \nearrow_{0}\right\}-\#\left\{\searrow_{0}^{\circ}\right\},
$$

the difference between the number of two types of configurations in $\mathcal{H}(\mathcal{P})$. These configurations are said to be $\wedge$-triples and $\vee$-triples, respectively. Using this interpretation, one obtains the following result.

Proposition 2.6. Suppose $\tilde{\mathcal{P}}$ is a distributive lattice and $\mathcal{P} \subset \tilde{\mathcal{P}}$ a subposet such that if $I \in \mathcal{P}$ and $I^{\prime} \preccurlyeq I\left(I^{\prime} \in \tilde{\mathcal{P}}\right)$, then $I^{\prime} \in \mathcal{P}$. Then $\mathcal{D}_{\mathcal{P}}(1) \leqslant 0$. Furthermore, $\mathcal{D}_{\mathcal{P}}(1)=0$ if and only if $\mathcal{P}$ is a distributive lattice if and only if $\mathcal{D}_{\mathcal{P}} \equiv 0$.

Proof. Here each $\wedge$-triple can be completed to a diamond inside $\mathcal{P}$, i.e., the configuration of the form ' $\vee$ '. This provides an injection of the set of $\wedge$ triples to the set of $\vee$-triples. If this is a bijection, i.e., each $\vee$-triple can be included in a diamond, then $\mathcal{P}$ has a unique maximal element. Hence $\mathcal{P}$ is a distributive lattice and $\mathcal{D}_{\mathcal{P}}=0$.

In the following sections, we consider the polynomials $\hat{\mathcal{K}}_{\mathcal{P}}, \check{\mathcal{K}}_{\mathcal{P}}$, and $\mathcal{D}_{\mathcal{P}}$ for the posets described in the Introduction.

\section{Covering polynomials for the root systems}

Our main reference for root systems and their properties is [3]. Let $\Delta$ be a root system in an $n$-dimensional real euclidean vector space $V$. Choose a subsystem of positive roots $\Delta^{+}$with the corresponding set of simple roots $\Pi=\left\{\alpha_{1}, \ldots, \alpha_{n}\right\}$. Write $\theta$ for the highest root in $\Delta^{+}$and $h$ for the Coxeter number. The standard root order ' $\preccurlyeq$ ' in $\Delta^{+}$is determined by the condition that $\gamma$ covers $\mu$ if and only if $\gamma-\mu \in \Pi$. Then $\theta$ is the unique maximal element of $\Delta^{+}$. Our goal is to compute both covering polynomials for $\left(\Delta^{+}, \preccurlyeq\right)$. In view of Lemma 2.1, it suffices to consider the irreducible root systems.

If $\Delta$ is reduced and irreducible, then $\mathfrak{g}$ is the corresponding simple Lie algebra. If $\theta=\sum_{i=1}^{n} m_{i} \alpha_{i}$, then in this case one also has $\sum_{i} m_{i}=h-1[3$, Ch. VI $\S 1.11$, Prop. 31].

In what follows, $\left[q^{m}\right] \mathcal{F}$ stands for the coefficient of $q^{m}$ in the polynomial $\mathcal{F}(q)$.

Theorem 3.1. Let $\Delta$ be an irreducible root system of rank $n$. Then

(i) $\operatorname{deg} \hat{\mathcal{K}}_{\Delta^{+}} \leqslant 3$ and $\operatorname{deg} \check{\mathcal{K}}_{\Delta^{+}} \leqslant 3$; 
(ii) $\left[q^{3}\right] \hat{\mathcal{K}}_{\Delta^{+}}=\left[q^{3}\right] \check{\mathcal{K}}_{\Delta^{+}}$;

(iii) if $\Delta$ is reduced, then $[q] \hat{\mathcal{K}}_{\Delta^{+}}=h-1-n$;

(iv) if $\Delta$ is simply-laced, then $[q] \hat{\mathcal{K}}_{\Delta^{+}}=\left[q^{3}\right] \hat{\mathcal{K}}_{\Delta^{+}}$;

(v) $\mathcal{D}_{\Delta^{+}}(q) \equiv n-1$.

Proof. We provide a uniform proof for parts (i) and (ii) only in the simplylaced case. The remaining cases (including the non-reduced root system $\mathbf{B C}_{n}$, see below) can be handled in a case-by-case fashion.

(i) For $\hat{\mathcal{K}}_{\Delta^{+}}$, one has to show that there are at most 3 simple roots that can be subtracted from a positive root. Suppose $\gamma \in \Delta^{+}$and $\gamma-\alpha_{i} \in \Delta^{+}$for $\alpha_{i} \in \Pi$ and $i=1,2, \ldots, k$. If $\left(\alpha_{1}, \alpha_{2}\right) \neq 0$, then these two adjacent simple roots generate the root system of type $\mathbf{A}_{2}$. Furthermore, $\gamma$ is the highest weight in the adjoint $\mathbf{A}_{2}$-module inside $\mathfrak{g}$. Therefore the weight $\gamma-\alpha_{1}-\alpha_{2}$ has multiplicity two. This is only possible if $\gamma=\alpha_{1}+\alpha_{2}$ and hence $k=2$. This also proves that if $k \geqslant 3$, then all simple roots that can be subtracted from $\gamma$ are pairwise orthogonal. In this situation, it was shown in [13, Corollary 3.3] that $k \leqslant 3$.

The argument for $\check{\mathcal{K}}_{\Delta^{+}}$is similar.

(ii) Suppose that $\kappa(\gamma)=3$, and let $\alpha_{1}, \alpha_{2}, \alpha_{3}$ be the corresponding simple roots. As is shown in part (i), these roots are pairwise orthogonal. Therefore $\gamma-\alpha_{1}-\alpha_{2}-\alpha_{3} \in \Delta^{+}$and the mapping $\gamma \mapsto\left(\gamma-\alpha_{1}-\alpha_{2}-\alpha_{3}\right)$ sets up a bijection between $\left\{\gamma \in \Delta^{+} \mid \kappa(\gamma)=3\right\}$ and $\left\{\gamma \in \Delta^{+} \mid \iota(\gamma)=3\right\}$.

(iii) If $\gamma=\sum_{j} a_{j} \alpha_{j} \in \Delta^{+}$, then $\left[\gamma: \alpha_{j}\right]:=a_{j}$ is called the $\alpha_{j}$-height of $\gamma$. Suppose that $\kappa(\gamma)=1$, i.e., there is a unique $\alpha_{i} \in \Pi$ such that $\gamma-\alpha_{i} \in \Delta^{+}$. Let $\mathfrak{l}_{i}$ denote the semisimple subalgebra of $\mathfrak{g}$ whose set of simple roots if $\Pi \backslash\left\{\alpha_{i}\right\}$. All the roots with a fixed $\alpha_{i}$-height form the set of weights of a simple $\mathfrak{l}_{i}$-submodule inside $\mathfrak{g}[10,2.1]$. Therefore, for each value $\geqslant 2$ of $\alpha_{i}$-height, there is a unique root $\gamma$ with the property that $\kappa(\gamma)=1$. Hence, the number of positive roots $\gamma$ such that $\alpha_{i}$ is the only simple root that can be subtracted from $\gamma$ equals $m_{i}-1$. Thus, $\#\left\{\gamma \in \Delta^{+} \mid \kappa(\gamma)=1\right\}=$ $\sum_{i=1}^{n}\left(m_{i}-1\right)=h-1-n$.

(iv) As is well known, the number of positive roots is $\hat{\mathcal{K}}_{\Delta^{+}}(1)=n h / 2$. By [16, Theorem 1.1], the number of edges of $\mathcal{H}\left(\Delta^{+}\right)$equals $n(h-2)$ in the simply laced case. That is, $\hat{\mathcal{K}}_{\Delta^{+}}^{\prime}(1)=n(h-2)$. Writing $\hat{\mathcal{K}}_{\Delta^{+}}(q)=n+a q+$ $b q^{2}+c q^{3}$ and using the above two equalities, we obtain $a=c$.

(v) By parts (i) and (ii), $\operatorname{deg}\left(\hat{\mathcal{K}}_{\Delta^{+}}-\check{\mathcal{K}}_{\Delta^{+}}\right) \leqslant 2$. It is also clear that $\left[q^{0}\right]\left(\hat{\mathcal{K}}_{\Delta^{+}}-\mathcal{K}_{\Delta^{+}}\right)=n-1$. Since $(q-1)^{2}$ divides this polynomial, the quotient must be $n-1$.

Formulae of the theorem, together with known values $\hat{\mathcal{K}}_{\Delta^{+}}(1)$ and $\hat{\mathcal{K}}_{\Delta^{+}}^{\prime}(1)$, allow us to write down a closed formula for $\hat{\mathcal{K}}_{\Delta^{+}}$in the simplylaced case: 
Corollary 3.2. In the A-D-E case, we have

$$
\hat{\mathcal{K}}_{\Delta^{+}}(q)=1+(h-1-n) q+\left(\frac{n h}{2}+n-2 h+2\right) q^{2}+(h-1-n) q^{3} .
$$

It is not hard to compute covering polynomials for the posets $\Delta^{+}$in the remaining cases, see Table 1.

Table 1: The covering polynomials for the root systems

\begin{tabular}{cll}
\hline$\Delta$ & $\hat{\mathcal{K}}_{\Delta^{+}}(q)$ & $\check{\mathcal{K}}_{\Delta^{+}}(q)$ \\
\hline $\mathbf{A}_{n}$ & $n+\left(\begin{array}{c}n \\
2\end{array}\right) q^{2}$ & $1+(2 n-2) q+\left(\begin{array}{c}n-1 \\
2\end{array}\right) q^{2}$ \\
$\mathbf{B}_{n}, \mathbf{C}_{n}$ & $n+(n-1) q+(n-1)^{2} q^{2}$ & $1+(3 n-3) q$ \\
& & $+(n-1)(n-2) q^{2}$ \\
$\mathbf{B C}_{n}$ & $n+n q+n(n-1) q^{2}$ & $1+(3 n-2) q+(n-1)^{2} q^{2}$ \\
$\mathbf{D}_{n}$ & $n+(n-3) q+\left(\left(\begin{array}{c}n \\
2\end{array}\right)+\left(\begin{array}{c}n-3 \\
2\end{array}\right)\right) q^{2}$ & $1+(3 n-5) q+\left(\left(\begin{array}{c}n-1 \\
2\end{array}\right)+\left(\begin{array}{c}n-3 \\
2\end{array}\right)\right) q^{2}$ \\
& $+(n-3) q^{3}$ & $+(n-3) q^{3}$ \\
$\mathbf{E}_{6}$ & $6+5 q+20 q^{2}+5 q^{3}$ & $1+15 q+15 q^{2}+5 q^{3}$ \\
$\mathbf{E}_{7}$ & $7+10 q+36 q^{2}+10 q^{3}$ & $1+22 q+30 q^{2}+10 q^{3}$ \\
$\mathbf{E}_{8}$ & $8+21 q+70 q^{2}+21 q^{3}$ & $1+35 q+63 q^{2}+21 q^{3}$ \\
$\mathbf{F}_{4}$ & $4+7 q+12 q^{2}+q^{3}$ & $1+13 q+9 q^{2}+q^{3}$ \\
$\mathbf{G}_{2}$ & $2+3 q+q^{2}$ & $1+5 q$ \\
\hline
\end{tabular}

Let $\varepsilon_{1}, \ldots, \varepsilon_{n}$ be an orthonormal basis for $V$. Recall that

$$
\begin{aligned}
& \Delta\left(\mathbf{B}_{n}\right)=\left\{ \pm \varepsilon_{i} \pm \varepsilon_{j}(1 \leqslant i<j \leqslant n), \pm \varepsilon_{i}(1 \leqslant i \leqslant n)\right\} \\
& \Delta\left(\mathbf{C}_{n}\right)=\left\{ \pm \varepsilon_{i} \pm \varepsilon_{j}(1 \leqslant i<j \leqslant n), \pm 2 \varepsilon_{i}(1 \leqslant i \leqslant n)\right\}
\end{aligned}
$$

and the unique non-reduced irreducible root system $\mathbf{B} \mathbf{C}_{n}$ is the union of these two, i.e.,

$$
\Delta\left(\mathbf{B C}_{n}\right)=\left\{ \pm \varepsilon_{i} \pm \varepsilon_{j}(1 \leqslant i<j \leqslant n), \pm \varepsilon_{i}, \pm 2 \varepsilon_{i}(1 \leqslant i \leqslant n)\right\}
$$

The following observation reduces many questions about $\mathbf{B C}_{n}$ to $\mathbf{B}_{n+1}$ or $\mathbf{C}_{n+1}$.

Lemma 3.3. The poset of positive roots for $\mathbf{B C}_{n}$ is isomorphic to the subposet of non-simple positive roots for $\mathbf{B}_{n+1}$ or $\mathbf{C}_{n+1}$. The posets $\Delta^{+}\left(\mathbf{B}_{n+1}\right)$ and $\Delta^{+}\left(\mathbf{C}_{n+1}\right)$ are isomorphic. 
Proof. An order-preserving bijection between $\Delta^{+}\left(\mathbf{B C}_{n}\right)$ and either $\Delta^{+}\left(\mathbf{B}_{n+1}\right) \backslash \Pi$ or $\Delta^{+}\left(\mathbf{C}_{n+1}\right) \backslash \Pi$ is given as follows:

$$
\begin{aligned}
& \begin{array}{lll}
\mathbf{C}_{n+1} & \mathbf{B C}_{n} & \mathbf{B}_{n+1}
\end{array} \\
& \varepsilon_{i}-\varepsilon_{j+1} \longleftrightarrow \varepsilon_{i}-\varepsilon_{j} \longmapsto \quad \varepsilon_{i}-\varepsilon_{j+1} \quad(1 \leqslant i<j \leqslant n) \\
& \varepsilon_{i}+\varepsilon_{j} \longleftrightarrow \varepsilon_{i}+\varepsilon_{j} \longmapsto \varepsilon_{i}+\varepsilon_{j+1} \quad(1 \leqslant i<j \leqslant n) \\
& \varepsilon_{i}+\varepsilon_{n+1} \longleftrightarrow \varepsilon_{i} \longmapsto \varepsilon_{i} \quad(1 \leqslant i \leqslant n) \\
& 2 \varepsilon_{i} \longleftrightarrow \quad 2 \varepsilon_{i} \longmapsto \quad \varepsilon_{i}+\varepsilon_{i+1} \quad(1 \leqslant i \leqslant n)
\end{aligned}
$$

It is easily seen that this extends to an isomorphism between $\Delta^{+}\left(\mathbf{B}_{n+1}\right)$ and $\Delta^{+}\left(\mathbf{C}_{n+1}\right)$.

Example 3.4. Consider two modifications of $\Delta^{+}$.

1. Replace $\Delta^{+}$with $\widetilde{\Delta}^{+}=\Delta^{+} \cup\{0\}$, where $\{0\}$ is regarded as the unique minimal element in this new poset. Hence $\mathcal{H}\left(\widetilde{\Delta}^{+}\right)$gains $n$ new edges connecting $\{0\}$ with the simple roots. Therefore

$$
\begin{aligned}
& \hat{\mathcal{K}}_{\widetilde{\Delta}^{+}}(q)=\hat{\mathcal{K}}_{\Delta^{+}}(q)+n(q-1)+1, \\
& \check{\mathcal{K}}_{\widetilde{\Delta}^{+}}(q)=\check{\mathcal{K}}_{\Delta^{+}}(q)+q^{n} .
\end{aligned}
$$

It follows that

$$
\mathcal{D}_{\widetilde{\Delta}^{+}}(q)=\mathcal{D}_{\Delta^{+}}(q)-\frac{q^{n}-n q+n-1}{(q-1)^{2}}=-\left(q^{n-2}+2 q^{n-3}+\cdots+(n-2) q\right) .
$$

2. Assume that $n \geqslant 2$ and consider $\Delta^{+} \backslash \Pi$ as subposet of $\Delta^{+}$. Then the minimal elements of $\Delta^{+} \backslash \Pi$ are the roots of height 2. Here we obtain $\hat{\mathcal{K}}_{\Delta^{+} \backslash \Pi}(q)=\hat{\mathcal{K}}_{\Delta^{+}}(q)-(n-1) q^{2}-1$. But formulae for $\check{\mathcal{K}}$ depends on the presence of a branching node in the Dynkin diagram, i.e., on the presence of a simple root which is covered by three roots. More precisely,

$$
\begin{aligned}
& \check{\mathcal{K}}_{\Delta^{+} \backslash \Pi}(q) \\
& =\check{\mathcal{K}}_{\Delta^{+}}(q)- \begin{cases}(n-2) q^{2}+2 q, & \text { if } \Delta^{+} \text {does not have a branching node; } \\
q^{3}+(n-4) q^{2}+3 q, & \text { if } \Delta^{+} \text {has a branching node. }\end{cases}
\end{aligned}
$$

Then

$$
\mathcal{D}_{\Delta^{+} \backslash \Pi}(q)= \begin{cases}n-2, & \text { if } \Delta^{+} \text {does not have a branching node; } \\ q+n-2, & \text { if } \Delta^{+} \text {has a branching node. }\end{cases}
$$

Thus, the deviation polynomial of $\Delta^{+}, \Delta^{+} \cup\{0\}$, and $\Delta^{+} \backslash \Pi$ always has the nonzero coefficients of the same sign. 


\section{Covering polynomials for the poset of $a d$-nilpotent ideals}

Let $\mathfrak{g}$ be the simple complex Lie algebra corresponding to $\Delta$ (if $\Delta$ is reduced). Fix a triangular decomposition $\mathfrak{g}=\mathfrak{u} \oplus \mathfrak{t} \oplus \mathfrak{u}^{-}$, where $\mathfrak{t}$ is a Cartan subalgebra and the set of $\mathfrak{t}$-roots in $\mathfrak{u}$ is $\Delta^{+}$. Then $\mathfrak{b}=\mathfrak{t} \oplus \mathfrak{u}$ is the fixed Borel subalgebra.

An ad-nilpotent ideal of $\mathfrak{b}$ is a subspace $\mathfrak{c} \subset \mathfrak{u}$ such that $[\mathfrak{b}, \mathfrak{c}] \subset \mathfrak{c}$. Then $\mathfrak{c}$ is a sum of certain root spaces in $\mathfrak{u}, \mathfrak{c}=\bigoplus_{\gamma \in I} \mathfrak{g}_{\gamma}$. Here $I$ is necessarily an upper ideal of $\Delta^{+}$, and this shows that the poset of $a d$-nilpotent ideals of $\mathfrak{b}$ is isomorphic to the poset of upper ideals of $\Delta^{+}$. It will be denoted by $\mathfrak{A} \mathfrak{d}$ or $\mathfrak{A} \mathfrak{d}(\mathfrak{g})$. If $I \in \mathfrak{A} \mathfrak{d}$ is considered as a subset of $\Delta^{+}$, then $\kappa(I)=\# \min (I)$ and $\iota(I)=\# \max \left(\Delta^{+} \backslash I\right)$. The elements of $\min (I)$ are called generators of $I$. For $\gamma \in \max \left(\Delta^{+} \backslash I\right)$, the passage $I \mapsto I \cup\{\gamma\}$ is called an extension of $I$. Thus, $\kappa(I)$ (resp. $\iota(I)$ ) is the number of generators (resp. extensions) of $I$.

Recall that $\mathfrak{A} \mathfrak{d} \simeq J^{*}\left(\Delta^{+}\right)$and $\mathcal{K}_{\mathfrak{A d}}$ is the covering polynomial of $\mathfrak{A} \mathfrak{d}$. By Theorem 2.2, $\left[q^{k}\right] \mathcal{K}_{\mathfrak{A} \mathfrak{d}}$ equals the number of $k$-element antichains in $\Delta^{+}$. Here $\operatorname{deg} \mathcal{K}_{\mathfrak{A} \mathfrak{d}}=$ rk $\Delta=n$. Explicit formulae for $\mathcal{K}_{\mathfrak{A d}}$ for all simple Lie algebras $\mathfrak{g}$ can be found e.g. in [15, Section 6]. The polynomials $\mathcal{K}_{\mathfrak{A} \mathfrak{d}}$ occur in various contexts, see $[1,2,15,19]$. There is a uniform expression (and proof) for the number of all $a d$-nilpotent ideals, i.e., $\mathcal{K}_{\mathfrak{A d}}(1)$, see [5]. Since $\mathcal{K}_{\mathfrak{A O}}$ is palindromic, $\mathcal{K}_{\mathfrak{A} \mathfrak{d}}^{\prime}(1)=\frac{n}{2} \mathcal{K}_{\mathfrak{A d}}(1)$, which yields the expression for the number of edges in $\mathcal{H}(\mathfrak{A} \mathfrak{d})$, see [16]. The coefficients of $\mathcal{K}_{\mathfrak{A d}}$ are of great interest; for instance, for $\Delta$ of type $\mathbf{A}_{n}$, one obtains the classical Narayana numbers. But no uniform approach to describing the coefficients of $\mathcal{K}_{\mathfrak{A d}}$ is known. For future use, we record the relation between the number of vertices and edges in $\mathcal{H}(\mathfrak{A} \mathfrak{d})$ :

$$
\frac{\# \mathcal{E}(\mathfrak{A} \mathfrak{d})}{\# \mathfrak{A} \mathfrak{d}}=\frac{\mathcal{K}_{\mathfrak{A} \mathfrak{d}}^{\prime}(1)}{\mathcal{K}_{\mathfrak{A} \mathfrak{d}}(1)}=\frac{n}{2}=\frac{\#\left(\Delta^{+}\right)}{h} .
$$

There is no Lie algebra associated with the root system $\mathbf{B} \mathbf{C}_{n}$, but one can still consider the poset of upper ideals in $\Delta^{+}\left(\mathbf{B C}_{n}\right)$, denoted by $\mathfrak{A} \mathfrak{d}\left(\mathbf{B C}_{n}\right)$. By Lemma 3.3, \#( $\left.\mathfrak{A} \mathfrak{d}\left(\mathbf{B C}_{n}\right)\right)$ equals to the number of upper ideals in $\Delta^{+}\left(\mathbf{B}_{n+1}\right) \backslash \Pi$. The latter is known to be equal to $\left(\begin{array}{c}2 n+1 \\ n\end{array}\right)[21]$.

Proposition 4.1. The covering polynomial of $\mathfrak{A d}\left(\mathbf{B C}_{n}\right)$ is $\sum_{k \geqslant 0}\left(\begin{array}{c}n \\ k\end{array}\right)\left(\begin{array}{c}n+1 \\ k\end{array}\right) q^{k}$.

Proof. The poset $\Delta^{+}\left(\mathbf{B C}_{n}\right)$ is isomorphic to the trapezoidal poset $\mathcal{T}(n, n+$ 1) considered by Stembridge [23]. Therefore, the coefficient of $q^{k}$ in the covering polynomial of $\mathfrak{A} \mathfrak{d}\left(\mathbf{B C}_{n}\right)$ equals the number of $k$-element antichains in $\mathcal{T}(n, n+1)$. By [23, Theorem 5.4], the latter is the same as the number of $k$-element antichains in the rectangular poset $\mathcal{R}(n, n+1)$. It is easily seen that the number of $k$-element antichains in $\mathcal{R}(n, n+1)$ is $\left(\begin{array}{l}n \\ k\end{array}\right)\left(\begin{array}{c}n+1 \\ k\end{array}\right)$. 
Unlike the covering polynomial for the poset of upper ideals in a reduced root system, this polynomial is not palindromic.

Corollary 4.2. The number of edges of $\mathcal{H}\left(\mathfrak{A d}\left(\mathbf{B C}_{n}\right)\right)$ is equal to $n\left(\begin{array}{c}2 n \\ n\end{array}\right)=$ $(n+1)\left(\begin{array}{c}2 n \\ n+1\end{array}\right)$.

Proof.

$$
\begin{aligned}
& \left.\frac{d}{d q}\left(\sum_{k \geqslant 0}\left(\begin{array}{l}
n \\
k
\end{array}\right)\left(\begin{array}{c}
n+1 \\
k
\end{array}\right) q^{k}\right)\right|_{q=1} \\
& \quad=\sum_{k \geqslant 0} k\left(\begin{array}{l}
n \\
k
\end{array}\right)\left(\begin{array}{c}
n+1 \\
k
\end{array}\right)=(n+1) \sum_{k \geqslant 0}\left(\begin{array}{c}
n \\
k-1
\end{array}\right)\left(\begin{array}{l}
n \\
k
\end{array}\right)=(n+1)\left(\begin{array}{c}
2 n \\
n+1
\end{array}\right) .
\end{aligned}
$$

The poset $\mathfrak{A} \mathfrak{d}\left(\mathbf{B C}_{n}\right)$ can be regarded as a particular case of the following series of examples. An ad-nilpotent ideal $\mathfrak{c}$ is said to be strictly positive, if $\mathfrak{c} \subset[\mathfrak{u}, \mathfrak{u}]$. The combinatorial counterpart is that an upper ideal $I \subset \Delta^{+}$is strictly positive, if $I \cap \Pi=\varnothing$. The corresponding sub-poset of $\mathfrak{A} \mathfrak{d}$ is denoted by $\mathfrak{A d}_{0}$ or $\mathfrak{A d}_{0}(\mathfrak{g})$. Clearly, $\mathfrak{A d}_{0} \simeq J^{*}\left(\Delta^{+} \backslash \Pi\right)$ is a distributive lattice.

By Lemma 3.3, $\mathfrak{A} \mathfrak{d}\left(\mathbf{B C}_{n}\right) \simeq \mathfrak{A d}_{0}\left(\mathbf{B}_{n+1}\right) \simeq \mathfrak{A d}_{0}\left(\mathbf{C}_{n+1}\right)$. This prompts a natural question about $\mathfrak{A d}_{0}(\mathfrak{g})$ for the other root systems (simple Lie algebras). A general formula for $\# \mathfrak{A d}_{0}(\mathfrak{g})$, i.e., for $\mathcal{K}_{\mathfrak{A} \mathfrak{d}_{0}(\mathfrak{g})}(1)$, is found by Sommers [21]. In our setting, we are interested in the covering polynomial $\mathcal{K}_{\mathfrak{A d}_{0}(\mathfrak{g})}$. The answer for $\mathfrak{A d}_{0}\left(\mathbf{B}_{n+1}\right)$ and $\mathfrak{A d}_{0}\left(\mathbf{C}_{n+1}\right)$ is given in Proposition 4.1. The case of $\mathfrak{g}=\mathfrak{s l}_{n+1}$ is easy, because $\Delta^{+}\left(\mathbf{A}_{n}\right) \backslash \Pi \simeq \Delta^{+}\left(\mathbf{A}_{n-1}\right)$ and hence $\mathcal{K}_{\mathfrak{A d}_{0}\left(\mathbf{A}_{n}\right)}=\mathcal{K}_{\mathfrak{A d}\left(\mathbf{A}_{n-1}\right)}$. The exceptional root systems can be handled directly. For $\mathbf{D}_{n}$, the answer is not easy to obtain.

Conjecturally, $\mathcal{K}_{\mathfrak{A d}_{0}}$ can be expressed via Chapoton's $\mathcal{F}$-triangle as follows. Let $C_{k, l}$ be the set of cones in the cluster complex of $\Delta$ spanned by $k$ positive roots and $l$ negative simple roots, and $f_{k, l}=\#\left(C_{k, l}\right)$. Here $f_{k, l}=0$ if $k+l>n$. Define the $\mathcal{F}$-triangle by its generating function

$$
\mathcal{F}(\Delta)=\mathcal{F}(x, y)=\sum_{k, l \geqslant 0} f_{k, l} x^{k} y^{l} .
$$

We refer to [7] for relevant definitions and other background. Then we conjecture that

$$
\mathcal{K}_{\mathfrak{A d}_{0}}(q)=\sum_{k \geqslant 0} f_{k, 0} q^{k}(1-q)^{n-k}=(1-q)^{n} \mathcal{F}\left(\frac{q}{1-q}, 0\right)
$$


or

$$
\mathcal{K}_{\mathfrak{A d}_{0}}(q)=\sum_{k, l \geqslant 0} f_{k, l}(-1)^{l}(q-1)^{n-k}=(q-1)^{n} \mathcal{F}\left(\frac{1}{q-1},-1\right) .
$$

This gives the correct formula for $\mathcal{K}_{\mathfrak{A d}_{0}}(q)$ whenever we can verify it. (Explicit formulae for the $\mathcal{F}$-triangle can be found in [6] or [12].) We also notice that the coefficients of $\mathcal{K}_{\mathfrak{A d}_{0}}(q)$ give the "very positive $H$-vector" in Chapoton's terminology in [6]. For $\mathbf{D}_{n}$, this yields the following conjectural expression:

$$
\left[q^{k}\right] \mathcal{K}_{\mathfrak{A d}_{0}\left(\mathbf{D}_{n}\right)}=\left(\left(\begin{array}{c}
n-1 \\
k
\end{array}\right)\right)^{2}+\frac{k-2}{n-1}\left(\begin{array}{c}
n-1 \\
k
\end{array}\right)\left(\begin{array}{c}
n-1 \\
k-1
\end{array}\right)
$$

The information for the exceptional Lie algebras is gathered in Table 2.

Table 2: The covering polynomials for $\mathfrak{A d}_{0}(\mathfrak{g}), \mathfrak{g}$ being exceptional

\begin{tabular}{ll}
\hline$\Delta$ & \multicolumn{1}{c}{$\mathcal{K}_{\mathfrak{A d}_{0}}(q)$} \\
\hline $\mathbf{E}_{6}$ & $1+30 q+135 q^{2}+175 q^{3}+70 q^{4}+7 q^{5}$ \\
$\mathbf{E}_{7}$ & $1+56 q+420 q^{2}+952 q^{3}+770 q^{4}+216 q^{5}+16 q^{6}$ \\
$\mathbf{E}_{8}$ & $1+112 q+1323 q^{2}+4774 q^{3}+6622 q^{4}+3696 q^{5}+770 q^{6}+44 q^{7}$ \\
$\mathbf{F}_{4}$ & $1+20 q+35 q^{2}+10 q^{3}$ \\
$\mathbf{G}_{2}$ & $1+4 q$ \\
\hline
\end{tabular}

Using these data and above information for the classical series, we obtain:

$$
\frac{\# \mathcal{E}\left(\mathfrak{A d}_{0}\right)}{\#\left(\mathfrak{A d}_{0}\right)}=\frac{\mathcal{K}_{\mathfrak{A d}_{0}}^{\prime}(1)}{\mathcal{K}_{\mathfrak{A d}_{0}}(1)}=\frac{n}{2} \cdot \frac{h-2}{h-1}=\frac{\#\left(\Delta^{+} \backslash \Pi\right)}{h-1}
$$

This equality has a striking similarity with (4.1), and it would be interesting to find a conceptual explanation for it. More precisely, one can suggest a general pattern behind (4.1) and (4.2). Let $\mathcal{L}$ be a graded poset such that the maximal length of chains in $\mathcal{L}$ is $r-1$ (i.e., such a chain contains $r$ elements). These two equalities are manifestations of the following phenomenon:

For some "good" graded posets $\mathcal{L}$, one has

$$
\frac{\# \mathcal{E}\left(J^{*}(\mathcal{L})\right)}{\# J^{*}(\mathcal{L})}=\frac{\# \mathcal{L}}{r+1}
$$

Besides $\Delta^{+}$and $\Delta^{+} \backslash \Pi$, the weight posets of some (finite-dimensional) representations of $\mathfrak{g}$ considered in [17] also have this property. 


\section{Covering polynomials for the poset of abelian ideals}

There is an interesting subposet of $\mathfrak{A} \mathfrak{d}$, where the two covering polynomials are different. An ad-nilpotent ideal $\mathfrak{c} \subset \mathfrak{b}$ is abelian if $[\mathfrak{c}, \mathfrak{c}]=0$. The combinatorial counterpart is that an upper ideal $I \subset \Delta^{+}$is said to be abelian, if $\gamma^{\prime}+\gamma^{\prime \prime} \notin \Delta^{+}$for each pair $\gamma^{\prime}, \gamma^{\prime \prime} \in I$. Let $\mathfrak{A} \mathfrak{b}=\mathfrak{A} \mathfrak{b}(\mathfrak{g})$ be the subposet of $\mathfrak{A} \mathfrak{d}$ consisting of all abelian ideals. Clearly $\mathfrak{A} \mathfrak{b}$ is a graded meet-semilattice. It follows that $\mathfrak{A b}$ is a (distributive) lattice if and only if there is a unique maximal abelian ideal, which happens only for $\mathbf{C}_{n}$ and $\mathbf{G}_{2}$. In all other cases the upper and lower covering polynomials are different.

Our next results rely on the relationship, due to D. Peterson, between the abelian ideals and the so-called minuscule elements of the affine Weyl group of $\mathfrak{g}$. Recall the necessary setup.

We have the real vector space $V=\oplus_{i=1}^{n} \mathbb{R} \alpha_{i}$ and ( , ) a $W$-invariant inner product on $V$.

$Q=\oplus_{i=1}^{n} \mathbb{Z} \alpha_{i} \subset V$ is the root lattice;

$Q^{+}=\left\{\sum_{i=1}^{n} m_{i} \alpha_{i} \mid m_{i}=0,1,2, \ldots\right\}$ is the monoid generated by the positive roots.

As usual, $\mu^{\vee}=2 \mu /(\mu, \mu)$ is the coroot for $\mu \in \Delta$ and $Q^{\vee}=\oplus_{i=1}^{n} \mathbb{Z} \alpha_{i}^{\vee}$ is the coroot lattice.

Letting $\widehat{V}=V \oplus \mathbb{R} \delta \oplus \mathbb{R} \lambda$, we extend the inner product ( , ) on $\widehat{V}$ so that $(\delta, V)=(\lambda, V)=(\delta, \delta)=(\lambda, \lambda)=0$ and $(\delta, \lambda)=1$. Then

$\widehat{\Delta}=\{\Delta+k \delta \mid k \in \mathbb{Z}\}$ is the set of affine (real) roots;

$\widehat{\Delta}^{+}=\Delta^{+} \cup\{\Delta+k \delta \mid k \geqslant 1\}$ is the set of positive affine roots;

$\widehat{\Pi}=\Pi \cup\left\{\alpha_{0}\right\}$ is the corresponding set of affine simple roots.

Here $\alpha_{0}=\delta-\theta$. For $\alpha_{i}(0 \leqslant i \leqslant n)$, let $s_{i}$ denote the corresponding reflection in $G L(\widehat{V})$. That is, $s_{i}(x)=x-\left(x, \alpha_{i}\right) \alpha_{i}^{\vee}$ for any $x \in \widehat{V}$. The affine Weyl group, $\widehat{W}$, is the subgroup of $G L(\widehat{V})$ generated by the reflections $s_{i}$, $i=0,1, \ldots, n$. If the index of $\alpha \in \widehat{\Pi}$ is not specified, then we merely write $s_{\alpha}$. The inner product (, ) on $\widehat{V}$ is $\widehat{W}$-invariant. The notation $\beta>0$ (resp. $\beta<0$ ) is a shorthand for $\beta \in \widehat{\Delta}^{+}$(resp. $\beta \in-\widehat{\Delta}^{+}$). The length function on $\widehat{W}$ with respect to $s_{0}, s_{1}, \ldots, s_{p}$ is denoted by $\ell$. For $w \in \widehat{W}$, we set $\mathcal{N}(w)=\left\{\nu \in \widehat{\Delta}^{+} \mid w(\nu)<0\right\}$. Then $\# \mathcal{N}(w)=\ell(w)$.

Definition 2 (D. Peterson). An element $w \in \widehat{W}$ is said to be minuscule, if $\mathcal{N}(w)=\{\delta-\gamma \mid \gamma \in I\}$ for some subset $I \subset \Delta$.

Then one can easily show that $I \subset \Delta^{+}, I$ is an abelian ideal, and this correspondence yields a bijection between the minuscule elements of $\widehat{W}$ and 
the abelian ideals. Furthermore, if $w$ is minuscule and $w^{-1}(\alpha)=-\mu+k \delta$ $(\alpha \in \widehat{\Pi}, \mu \in \Delta)$, then $k \geqslant-1$. (More generally, this holds for elements of $\widehat{W}$ corresponding to arbitrary ad-nilpotent ideals, see [4].) If $w$ is minuscule, then $I_{w}$ denotes the corresponding abelian ideal. Conversely, given $I \in \mathfrak{A} \mathfrak{b}$, then $w_{I}$ stands for the corresponding minuscule element. If $I \in \mathfrak{A} \mathfrak{b}$ and $\gamma \in \max \left(\Delta^{+} \backslash I\right)$, then the ideal $I^{\prime}=I \cup\{\gamma\}$ is not necessarily abelian. In this section, we are interested only in abelian extensions, i.e., those with abelian $I^{\prime}$.

Lemma 5.1. Suppose $w \in \widehat{W}$ is minuscule and $w^{-1}(\alpha)=-\mu+2 \delta$, where $\alpha \in \widehat{\Pi}$ and $\mu \in \Delta$. Then $\mu=\theta$.

Proof. We have $\alpha=w(2 \delta-\mu)=w(2 \delta-\theta)+w(\theta-\mu)$. Here $\theta-\mu \in Q^{+}$. Hence both $2 \delta-\theta$ and $\theta-\mu$ do not belong to $\mathcal{N}(w)$. Therefore if $\theta \neq \mu$, then one obtains a contradiction with the fact that $\alpha$ is simple.

For a minuscule $w$, consider the vector $\mathbf{k}=\mathbf{k}_{w}=\left(k_{0}, k_{1}, \ldots, k_{n}\right)$, where $k_{i}$ is defined by the equality $w^{-1}\left(\alpha_{i}\right)=-\mu_{i}+k_{i} \delta\left(\mu_{i} \in \Delta\right)$. Recall that $k_{i} \geqslant-1$ for all $i$.

Proposition 5.2. We have

(i) $k_{i} \leqslant 2$ for all $i$;

(ii) there is at most one index $i$ such that $k_{i}=2$. The corresponding simple root $\alpha_{i}$ is necessarily long.

(iii) $k_{0} \leqslant 1$; that is, $k_{0} \neq 2$.

Proof.

(i) If $w^{-1}\left(\alpha_{i}\right)=-\mu_{i}+k_{i} \delta$ and $k_{i} \geqslant 3$, then $w\left(2 \delta-\mu_{i}\right)=-\left(k_{i}-2\right) \delta+\alpha_{i}<$ 0 . Hence $w$ is not minuscule.

(ii) If $w^{-1}\left(\alpha_{i}\right)=-\mu_{i}+2 \delta$, then $\mu_{i}=\theta$ by Lemma 5.1. Hence such $i$ is unique and $\left\|\alpha_{i}\right\|=\|\theta\|$, i.e., $\alpha_{i}$ is long.

(iii) Suppose $w^{-1}\left(\alpha_{0}\right)=-\mu_{0}+2 \delta$. Then $\mu_{0}=\theta$ and $w(2 \delta-\theta)=\delta-\theta$. However, it is shown in [14, Prop. 2.5] that $w(2 \delta-\theta) \in \Delta^{+}$for any non-trivial minuscule element $w$.

We shall say that $\mathbf{k}_{w}$ is the shift vector of $w$ or $I_{w}$. If $w=w_{I}(I \in \mathfrak{A} \mathfrak{b})$, then we also write $\mathbf{k}_{I}$ for this vector.

Theorem 5.3. Let $\left(k_{0}, k_{1}, \ldots, k_{n}\right)$ be the shift vector of $I \in \mathfrak{A} \mathfrak{b}$. Then $\kappa(I)=\#\left\{i \mid k_{i}=-1\right\}$ and $\iota(I)=\#\left\{i \mid k_{i}=1\right\}$. 
Proof. 1. It is shown in [14, Theorem 2.2] that $\gamma \in I$ is a generator if and only if $w_{I}(\delta-\gamma)=-\alpha_{i} \in-\widehat{\Pi}$. That is, $k_{i}=-1$ for the corresponding coordinate $i$.

2. Suppose that $k_{i}=1$, i.e., $w_{I}^{-1}\left(\alpha_{i}\right)=-\mu_{i}+\delta$. Then $w_{I}\left(-\mu_{i}\right)=$ $\alpha_{i}-\delta<0$. As $w_{I}$ is minuscule, $\mu_{i}$ must be positive. Next, $\mathcal{N}\left(s_{i} w_{I}\right)=$ $\mathcal{N}\left(w_{I}\right) \cup\left\{w_{I}^{-1}\left(\alpha_{i}\right)\right\}$. Hence $s_{i} w_{I}$ is again minuscule, and the corresponding abelian ideal is $\tilde{I}=I \cup\left\{\mu_{i}\right\}$. Conversely, if $I \rightarrow I \cup\{\gamma\}$ is an abelian extension of $I$, then $w_{\tilde{I}}=s_{i} w_{I}$ for some $i \in\{0,1, \ldots, n\}$ and $w_{\tilde{I}}(\delta-\gamma)=-\alpha_{i}[14$, Theorem 2.4]. Then $w_{I}(\delta-\gamma)=\alpha_{i}$, i.e., $k_{i}=1$.

Since the minuscule elements ( $\sim$ abelian ideals) can be constructed recursively, we obtain, as a consequence of this theorem, a method to compute recursively the shift vector. One starts with the minuscule element $1 \in \widehat{W}$ (or the empty ideal). The corresponding shift vector is $\mathbf{k}_{1}=(1,0, \ldots, 0)$. The inductive step is to replace $w=w_{I}$ with $s_{i} w$ for some $i$. However one has to be careful while choosing $s_{i}$, otherwise $s_{i} w$ may fail to be minuscule.

Proposition 5.4. Suppose that $w \in \widehat{W}$ is minuscule. Then

- $s_{j} w$ is again minuscule if and only if $\left(\mathbf{k}_{w}\right)_{j}=1$.

- If $\left(\mathbf{k}_{w}\right)_{j}=1$, then $\mathbf{k}_{s_{j} w}=\mathbf{k}_{w}-(\text { the } j \text {-th column of the extended Cartan matrix of } \Delta)^{t}$.

Proof. The first claim is essentially proved in the second part of the above theorem. The second claim follows from the assumption $\left(\mathbf{k}_{w}\right)_{j}=1$ and the equalities:

$$
\left(s_{j} w\right)^{-1}\left(\alpha_{i}\right)=w^{-1}\left(\alpha_{i}\right)-\left(\alpha_{i}, \alpha_{j}^{\vee}\right) w^{-1}\left(\alpha_{j}\right) ; \quad i=0,1, \ldots, n .
$$

Recall that the extended Cartan matrix is the $(n+1) \times(n+1)$ matrix with entries $\left(\alpha_{i}, \alpha_{j}^{\vee}\right), 0 \leqslant i, j \leqslant n$.

Remark. Write $\theta=\sum_{i=1}^{n} c_{i} \alpha_{i}$ and set $c_{0}=1$. Then $\sum_{i=0}^{n} c_{i} \alpha_{i}=\delta$. Since $\delta$ is $\widehat{W}$-invariant, the definition of $k_{i}$ 's implies that $\sum_{i=0}^{n} c_{i} k_{i}=1$ for any shift vector. Hence $\mathbf{k}_{I}$ is fully determined by $k_{1}, \ldots, k_{n}$. Let $z=z_{I} \in V$ be the unique point such that $\left(z_{I}, \alpha_{i}\right)=k_{i}, i=1, \ldots, n$. Then $z \in Q^{\vee}$. (Again, this is true in the context of arbitrary ad-nilpotent ideals, see [5].) Note that $k_{0}=1-(z, \theta)$. The constraints of Proposition 5.2 show that $-1 \leqslant\left(z, \alpha_{i}\right) \leqslant 2$ for $i=1, \ldots, n$ and $0 \leqslant(z, \theta) \leqslant 2$. Furthermore, a stronger result is valid. It was shown by Kostant [11] that the mapping $I \in \mathfrak{A} \mathfrak{b} \mapsto z_{I} \in V$ sets up a bijection between the abelian ideals and the points $z \in Q^{\vee}$ such that $-1 \leqslant(z, \gamma) \leqslant 2$ for all $\gamma \in \Delta^{+}$.

Let $\Delta_{l}^{+}$denote the set of long positive roots and $\Pi_{l}:=\Delta_{l}^{+} \cap \Pi$. In [14], we constructed a disjoint partition of $\mathfrak{A}^{o} \mathfrak{b}:=\mathfrak{A} \mathfrak{b} \backslash\{\varnothing\}$ parametrised by $\Delta_{l}^{+}$. 
In other words, there is a natural surjective mapping $\tau: \stackrel{o}{\mathfrak{A} \mathfrak{b}} \rightarrow \Delta_{l}^{+}$. Given $I \in \mathfrak{A}^{o} \mathfrak{b}$ and the corresponding minuscule element $w \in \widehat{W}$, we set $\tau(I)=$ $w(2 \delta-\theta)$. By [14, Prop. 2.5], it is an element of $\Delta_{l}^{+}$. Then $\mathfrak{A b}_{\mu}=\tau^{-1}(\mu)$.

Remark. Using the above definition of the shift vector of an abelian ideal and Lemma 5.1, one observes that $\left(\mathbf{k}_{I}\right)_{i}=2$ if and only if $\tau(I)=\alpha_{i}$, i.e., $I \in \mathfrak{A b}_{\alpha_{i}}$.

One of the main results of [14] is that each $\mathfrak{A} \mathfrak{b}_{\mu}$ has a unique maximal and unique minimal element, and that the maximal elements of $\mathfrak{A b}$ are exactly the maximal elements of $\mathfrak{A b}_{\alpha}, \alpha \in \Pi_{l}$. Let $I \in \mathfrak{A}_{\mathfrak{l} \mathfrak{b}}$ and $\tau(I)=\mu$. By [14, Prop. 3.2], if $I \rightarrow I^{\prime}$ is an extension, then $\tau\left(I^{\prime}\right) \preccurlyeq \tau(I)$. Hence $I$ has an extension outside $\mathfrak{A b}_{\mu}$ only if $\mu \notin \Pi$. Now we make that analysis more precise by showing that the number of possible abelian extensions of $I \rightarrow I^{\prime}$ such that $I^{\prime} \notin \mathfrak{A b}_{\mu}$ depends only on $\mu$ and not on $I$.

Theorem 5.5. For any $\mu \in \Delta_{l}^{+} \backslash \Pi$ and any $I \in \mathfrak{A b}_{\mu}$, the number of abelian extensions $I \rightarrow I^{\prime}$ such that $I^{\prime} \notin \mathfrak{A b}_{\mu}$ equals the number of $\alpha \in \Pi$ such that $(\mu, \alpha)>0$. In particular, this number does not depend on $I$, and if $\Delta$ is simply laced, then it is equal to $\kappa(\mu)$.

Proof. 1. Suppose $(\alpha, \mu)>0$ and $\mu^{\prime}:=s_{\alpha}(\mu)=\mu-n_{\alpha} \alpha$. Here $n_{\alpha} \in \mathbb{N}$ and $n_{\alpha}=1$ if and only if $\alpha$ is long. Anyway, $\mu^{\prime \prime}:=\mu-\alpha$ is again a root, and we will work with the sum $\mu=\alpha+\mu^{\prime \prime}$. Then $w_{I}^{-1}\left(\mu^{\prime \prime}+\alpha\right)=2 \delta-\theta$ and, as shown in the proof of Theorem 2.6 in [14], one has $w_{I}^{-1}\left(\mu^{\prime \prime}\right)=\delta-\gamma^{\prime \prime}$ and $w_{I}^{-1}(\alpha)=\delta-\gamma^{\prime}$ for some $\gamma^{\prime}, \gamma^{\prime \prime}$ such that $\gamma^{\prime}+\gamma^{\prime \prime}=\theta$. This shows that $w=$ $s_{\alpha} w_{I}$ is minuscule, $I_{w}=I \cup\left\{\gamma^{\prime}\right\}$, and $\tau\left(I_{w}\right)=s_{\alpha} w_{I}(2 \delta-\theta)=s_{\alpha}(\mu)=\mu^{\prime}$.

2. Conversely, suppose $I \in \mathfrak{A b}_{\mu}$ and $I \rightarrow I^{\prime}$ is an extension. Then $I^{\prime}=$ $I \cup\{\gamma\}$ for some $\gamma \in \Delta^{+}$and $w_{I^{\prime}}=s_{\alpha} w_{I}$, where $\alpha \in \Pi$ is determined by the equality $w_{I}(\delta-\gamma)=\alpha$. The condition $\tau\left(I^{\prime}\right) \neq \mu$ means $s_{\alpha}(\mu) \neq \mu$, i.e., $(\alpha, \mu) \neq 0$. To compute the sign, we notice that $(\alpha, \mu)=\left(w_{I}^{-1}(\alpha), w_{I}^{-1}(\mu)\right)=$ $(\delta-\gamma, 2 \delta-\theta)=(\gamma, \theta)$, which cannot be negative.

Corollary 5.6. The ideals having a unique abelian extension are the following:

(a) $\varnothing$;

(b) The maximal elements of posets $\mathfrak{A b}_{\mu}$, where $\mu \notin \Pi$ and the inequality $(\alpha, \mu)>0$ holds for a unique $\alpha \in \Pi$;

(c) The ideals having a unique abelian extension inside $\mathfrak{A b}_{\alpha}, \alpha \in \Pi_{l}$.

It was noticed in [14] that each $\mathfrak{A b}_{\mu}$ is a minuscule poset, i.e., there is a simple Lie algebra $\mathfrak{l}$ and a parabolic subalgebra $\mathfrak{p} \subset \mathfrak{l}$ with abelian nilpotent 
radical $\mathfrak{p}^{\text {nil }}$ such that $\mathfrak{A} \mathfrak{b}_{\mu}$ is isomorphic to the poset of abelian $\mathfrak{p}$-ideals in $\mathfrak{p}^{\text {nil }}$. The construction of $\mathfrak{l}$ as a subalgebra of $\mathfrak{g}$ is given in [14, Section 5]. Since the structure of the minuscule posets is well known, Theorem 5.5 provides an effective tool for computing the lower covering polynomial in the small rank cases, e.g. for the exceptional Lie algebras.

We say that a root $\gamma \in \Delta^{+}$is commutative, if the upper ideal generated by $\gamma$ is abelian. Clearly, the set of commutative roots forms an upper ideal. A uniform description of this ideal (and its cardinality) is given in $[16$, Theorem 4.4]. In particular, if the Dynkin diagram has no branching node then the number of commutative roots is $n(n+1) / 2$. A trivial but useful remark is that any abelian ideal consists of commutative roots.

In the following assertion, we gather some information that is helpful in practical computations of the covering polynomials.

\section{Proposition 5.7.}

(i) $\left[q^{0}\right] \hat{\mathcal{K}}_{\mathfrak{A b}}=1,\left[q^{0}\right] \check{\mathcal{K}}_{\mathfrak{A} \mathfrak{b}}=\#\left(\Pi_{l}\right)$;

(ii) $[q] \hat{\mathcal{K}}_{\mathfrak{A} \mathfrak{b}}=$ the number of commutative roots;

(iii) $\operatorname{deg} \hat{\mathcal{K}}_{\mathfrak{A b}} \leqslant \operatorname{deg} \check{\mathcal{K}}_{\mathfrak{A} \mathfrak{b}}$. If these degrees are equal, say to $m$, then $\left[q^{m}\right] \hat{\mathcal{K}}_{\mathfrak{A b}} \leqslant\left[q^{m}\right] \mathcal{K}_{\mathfrak{A} \mathfrak{b}}$

(iv) $\hat{\mathcal{K}}_{\mathfrak{A} \mathfrak{b}}(1)=\check{\mathcal{K}}_{\mathfrak{A} \mathfrak{b}}(1)=2^{n}$;

(v) $\hat{\mathcal{K}}_{\mathfrak{A} \mathfrak{b}}^{\prime}(1)=\check{\mathcal{K}}_{\mathfrak{A} \mathfrak{b}}^{\prime}(1)=(n+1) 2^{n-2}$.

Proof.

(i) These are the numbers of minimal and maximal elements of $\mathfrak{A b}$.

(ii) Obvious.

(iii) Let $I$ be an abelian ideal with $m$ generators, say $\left\{\gamma_{1}, \ldots, \gamma_{m}\right\}$. Then $I \backslash\left\{\gamma_{1}, \ldots, \gamma_{m}\right\}$ has at least $m$ extensions. Then take $m=\operatorname{deg} \hat{\mathcal{K}}_{\mathfrak{A} \mathfrak{b}}$.

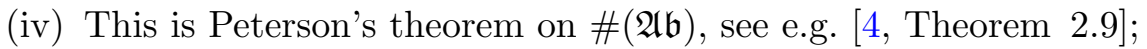

(v) This is the number of edges of $\mathcal{H}(\mathfrak{A} \mathfrak{b})$, which is computed in [16, Theorem 4.1].

Let $m$ be the maximal size of an antichain of commutative roots. Then $\operatorname{deg} \check{\mathcal{K}}_{\mathfrak{A} \mathfrak{b}} \leqslant m$. But this bound may not be sharp, since the ideal having a prescribed antichain as the set of either generators or minimal elements in the complement can be non-abelian. However, in case $\mathbf{E}_{7}$ and $\mathbf{E}_{8}$ this bound does give the exact value for both degrees, and we obtain $\operatorname{deg} \mathcal{K}_{\mathfrak{A} \mathfrak{b}\left(\mathbf{E}_{7}\right)}=$ $\operatorname{deg} \mathcal{K}_{\mathfrak{A} \mathfrak{b}\left(\mathbf{E}_{8}\right)}=4$, where $\mathcal{K}$ is either $\hat{\mathcal{K}}$ or $\check{\mathcal{K}}$.

Also, it follows from a result of Sommers [21, Theorem 6.4] that the number of generators of an abelian ideal is at most the maximal number of pairwise orthogonal roots in $\Pi$. This provides an upper bound for $\operatorname{deg} \hat{\mathcal{K}}_{\mathfrak{A} \mathfrak{b}}$. 
Since it is easy to find an abelian ideal with such a number of generators, one actually obtains the exact value of the degree. For instance, $\operatorname{deg} \hat{\mathcal{K}}_{\mathfrak{A b}\left(\mathbf{D}_{n}\right)}=$ $\left[\frac{n}{2}\right]+1$ and $\operatorname{deg} \hat{\mathcal{K}}_{\mathfrak{A} \mathfrak{b}\left(\mathbf{E}_{6}\right)}=3$. If $\operatorname{deg} \check{\mathcal{K}}_{\mathfrak{A} \mathfrak{b}} \leqslant 3$, then both covering polynomials can be computed using Proposition 5.7. This applies to $\mathbf{F}_{4}$ and $\mathbf{E}_{6}$. For $\mathbf{E}_{7}$ and $\mathbf{E}_{8}$, it suffices to determine one more value (or coefficient) of $\hat{\mathcal{K}}_{\mathfrak{A} \mathfrak{b}}$ and $\check{\mathcal{K}}_{\mathfrak{A b}}$.

Example 5.8. $\mathfrak{g}=\mathbf{E}_{8}$.

(1) We use Corollary 5.6 to compute the coefficient $[q] \check{\mathcal{K}}_{\mathfrak{A b}\left(\mathbf{E}_{8}\right)}$. Here the number of roots $\mu$ such that $\kappa(\mu)=1$ is 21 (see Section 3). The posets $\mathfrak{A b}_{\alpha_{i}}$, $\alpha_{i} \in \Pi$, have the cardinalities 1, 2, 3, 4, 5, 6, 8, 6. Furthermore, for $\mathbf{E}_{8}$, each poset $\mathfrak{A} \mathfrak{b}_{\mu}$ is totally ordered. Hence the contribution from part (c) of the corollary is $0+1+2+3+4+5+7+5=27$. Thus, $[q] \check{\mathcal{K}}_{\mathfrak{A} \mathfrak{b}\left(\mathbf{E}_{8}\right)}=1+21+27=49$.

(2) Since each $\mathfrak{A b}_{\mu}$ is a chain, any ideal $I$ has at most one extension inside its own poset $\mathfrak{A} \mathfrak{b}_{\mu}$. Because $\kappa(\mu) \leqslant 3$ for all $\mu \in \Delta^{+}$, Theorem 5.5 shows that

$$
\left[q^{4}\right] \check{\mathcal{K}}_{\mathfrak{A} \mathfrak{b}\left(\mathbf{E}_{8}\right)}=\sum_{\kappa(\mu)=3} \#\left(\mathfrak{A}_{\mu}\right)-1 .
$$

We have $\#\left\{\mu \in \Delta^{+} \mid \kappa(\mu)=3\right\}=\left[q^{3}\right] \hat{\mathcal{K}}_{\Delta^{+}\left(\mathbf{E}_{8}\right)}=21$. Of these 21 roots, there are

11 roots with $\#\left(\mathfrak{A b}_{\mu}\right)=1$ (these are exactly the roots $\mu$ with $(\theta, \mu) \neq 0$, see $[14,5.1]$;

5 roots with $\#\left(\mathfrak{A} \mathfrak{b}_{\mu}\right)=2$;

3 roots with $\#\left(\mathfrak{A b}_{\mu}\right)=3$;

2 roots with $\#\left(\mathfrak{A} \mathfrak{b}_{\mu}\right)=4$.

Hence $\left[q^{4}\right] \check{\mathcal{K}}_{\mathfrak{A} \mathfrak{b}\left(\mathbf{E}_{8}\right)}=17$.

(3) Then using Proposition 5.7(iv),(v), we compute $\check{\mathcal{K}}_{\mathfrak{A} \mathfrak{b}\left(\mathbf{E}_{8}\right)}(q)=8+$ $49 q+87 q^{2}+95 q^{3}+17 q^{4}$.

\section{Computing the covering polynomials for $\mathfrak{A} \mathfrak{b}(\mathfrak{g}), \mathfrak{g}$ being classical}

In this section, we prove four theorems for four classical series of simple Lie algebras (root systems). Our proofs are based on an explicit presentation of the upper ideal of commutative roots and understanding which ideals inside it are really abelian. To this end, one has to know the generators of all maximal abelian ideals, which are determined in [20] (see also [18, Table 1]).

Theorem 6.1. If $\mathfrak{g}=\mathfrak{s l}_{n+1}$, i.e., $\Delta$ is of type $\mathbf{A}_{n}$, then 
(i) $\hat{\mathcal{K}}_{\mathfrak{A b}}(q)=\sum_{k \geqslant 0}\left(\begin{array}{c}n+1 \\ 2 k\end{array}\right) q^{k}$;

(ii) $\check{\mathcal{K}}_{\mathfrak{A} \mathfrak{b}}(q)=\sum_{k \geqslant 0}\left(\left(\begin{array}{c}n \\ 2 k+1\end{array}\right)+\left(\begin{array}{c}n \\ 2 k-2\end{array}\right)\right) q^{k}$;

(iii) $\mathcal{D}_{\mathfrak{A} \mathfrak{b}}(q)=-\sum_{k \geqslant 0}\left(\begin{array}{c}n-1 \\ 2 k+1\end{array}\right) q^{k}$.

Proof. The formula for $\hat{\mathcal{K}}$ (but not for $\check{\mathcal{K}}$ !) is implicit in [18, Section 3]. We recall the necessary setup and then deduce the expressions (i) and (ii). Then part (iii) is obtained via formal manipulations.

For $\mathfrak{g}=\mathfrak{s l}_{n+1}$, each positive root is commutative. The root $\varepsilon_{i}-\varepsilon_{j} \in$ $\Delta^{+}\left(\mathfrak{s l}_{n+1}\right)$ is identified with the pair $(i, j)$. Suppose $\mathfrak{a} \in \mathfrak{A} \mathfrak{b}\left(\mathfrak{s l}_{n+1}\right)$ and $\kappa(\mathfrak{a})=k$. That is, $\mathfrak{a}$ has $k$ generators (minimal roots). If $\min (\mathfrak{a})=$ $\left\{\left(a_{1}, b_{1}\right), \ldots,\left(a_{k}, b_{k}\right)\right\}$, where $a_{1}<a_{2}<\cdots<a_{k}$, then we actually have $1 \leqslant a_{1}<a_{2}<\cdots<a_{k}<b_{1}<\cdots<b_{k} \leqslant n+1$. Thus, any $2 k$-element subset of $[1, n+1]$ gives rise to an abelian ideal with $\kappa(\mathfrak{a})=k$ and vice versa. This yields (i).

The edges of $\mathcal{H}(\mathfrak{A} \mathfrak{b})$ originating in $\mathfrak{a}$ bijectively correspond to the maximal roots $\gamma$ in $\Delta^{+} \backslash \mathfrak{a}$ such that $\{\gamma\} \cup \mathfrak{a}$ is again an abelian ideal. The set of such maximal roots always contains $\left\{\left(a_{1}+1, b_{2}-1\right), \ldots,\left(a_{k-1}+1, b_{k}-1\right)\right\}$; furthermore, if $a_{k}+1<b_{1}$, then two more roots are admissible: $\left(1, b_{1}-\right.$ $1),\left(a_{k}+1, n+1\right)$. From this we deduce that $\iota(\mathfrak{a})=k$ if and only if one of the following two conditions hold:

$\left(\nabla_{1}\right) \# \min (\mathfrak{a})=k+1$ and $a_{k+1}+1=b_{1}$.

$\left(\diamond_{2}\right) \# \min (\mathfrak{a})=k-1$ and $a_{k-1}+1<b_{1}$.

In case $\left(\nabla_{1}\right)$ the ideal is determined by a sequence of $2 k+1$ integers

$1 \leqslant a_{1}<\cdots<a_{k}<a_{k+1}=b_{1}-1<b_{2}-1<\cdots<b_{k+1}-1 \leqslant n$.

Hence there are $\left(\begin{array}{c}n \\ 2 k+1\end{array}\right)$ such possibilities.

In case $\left(\nabla_{2}\right)$ the ideal is determined by a sequence of $2 k-2$ integers

$1 \leqslant a_{1}<\cdots<a_{k-1}<b_{1}-1<b_{2}-1<\cdots<b_{k-1}-1 \leqslant n$.

Hence there are $\left(\begin{array}{c}n \\ 2 k-2\end{array}\right)$ such possibilities. This proves (ii).

(iii) It follows from parts (i) and (ii) that

$$
\begin{aligned}
\hat{\mathcal{K}}_{\mathfrak{A} \mathfrak{b}}(q)-\check{\mathcal{K}}_{\mathfrak{A b}}(q) & =-\sum_{k \geqslant 0}\left(\left(\begin{array}{c}
n \\
2 k+1
\end{array}\right)-\left(\begin{array}{c}
n+1 \\
2 k
\end{array}\right)+\left(\begin{array}{c}
n \\
2 k-2
\end{array}\right)\right) q^{k} \\
& =-\sum_{k \geqslant 0}\left(\left(\begin{array}{c}
n-1 \\
2 k+1
\end{array}\right)-2\left(\begin{array}{c}
n-1 \\
2 k-1
\end{array}\right)+\left(\begin{array}{c}
n-1 \\
2 k-3
\end{array}\right)\right) q^{k} \\
& =-(q-1)^{2} \sum_{k \geqslant 0}\left(\begin{array}{c}
n-1 \\
2 k+1
\end{array}\right) q^{k} .
\end{aligned}
$$


Theorem 6.2. If $\mathfrak{g}=\mathfrak{s p}_{2 n}$, i.e., $\Delta$ is of type $\mathbf{C}_{n}$, then $\hat{\mathcal{K}}_{\mathfrak{A b}}(q)=\check{\mathcal{K}}_{\mathfrak{A b}}(q)=$ $\sum_{k \geqslant 0}\left(\begin{array}{c}n+1 \\ 2 k\end{array}\right) q^{k}$.

Proof. The commutative roots are $\left\{\varepsilon_{i}+\varepsilon_{j} \mid 1 \leqslant i \leqslant j \leqslant n\right\}$. Since these roots form the unique maximal abelian ideal, the poset $\mathfrak{A b}\left(\mathfrak{s p}_{2 n}\right)$ is a distributive lattice and $\hat{\mathcal{K}}_{\mathfrak{A b}}(q)=\check{\mathcal{K}}_{\mathfrak{A b}}(q)$. The explicit form of this polynomials stems from the description given in [18, Section 3]: the ideals with $k$ generators (or with $k$ extensions) are in a bijection with the the sequences $1 \leqslant a_{1}<$ $a_{2}<\cdots<a_{k} \leqslant b_{1}<\cdots<b_{k} \leqslant n$. Hence there are $\left(\begin{array}{c}n+1 \\ 2 k\end{array}\right)$ possibilities for them.

The poset of commutative roots for $\mathfrak{s p}_{2 n}$ is represented by the triangular Ferrers diagram with row lengths $(n, n-1, \ldots, 1)$ (=triangle "of size $n$ "). In the following two theorems, such a triangle occurs as a subposet of the poset of commutative roots for $\mathfrak{s o}_{2 n+1}$ and $\mathfrak{s o}_{2 n}$, which allows us to exploit the formula of Theorem 6.2.

Theorem 6.3. If $\mathfrak{g}=\mathfrak{s o}_{2 n+1}$, i.e., $\Delta$ is of type $\mathbf{B}_{n}$, then

(i) $\hat{\mathcal{K}}_{\mathfrak{A} \mathfrak{b}}(q)=\sum_{k \geqslant 0}\left(\begin{array}{c}n+1 \\ 2 k\end{array}\right) q^{k}$;

(ii) $\check{\mathcal{K}}_{\mathfrak{A b}}(q)=\sum_{k \geqslant 0}\left(\left(\begin{array}{c}n-1 \\ 2 k+1\end{array}\right)+\left(\begin{array}{c}n \\ 2 k-1\end{array}\right)+\left(\begin{array}{c}n-1 \\ 2 k-2\end{array}\right)\right) q^{k}$;

(iii) $\mathcal{D}_{\mathfrak{A} \mathfrak{b}}(q)=-\sum_{k \geqslant 0}\left(\begin{array}{c}n-2 \\ 2 k+1\end{array}\right) q^{k}$.

Proof. Here the commutative roots are $\left\{\varepsilon_{i}+\varepsilon_{j} \mid 1 \leqslant i<j \leqslant n\right\} \cup\left\{\varepsilon_{1}-\varepsilon_{i} \mid\right.$ $2 \leqslant i \leqslant n\} \cup\left\{\varepsilon_{1}\right\}$.

Graphically, this set is represented by a skew Ferrers diagram with row lengths $(2 n-1, n-2, n-3, \ldots, 1)$. See the sample figure for $\Delta$ of type $\mathbf{B}_{6}$, where $\varepsilon_{i j}=\varepsilon_{i}-\varepsilon_{j}$ and $\bar{\varepsilon}_{i j}=\varepsilon_{i}+\varepsilon_{j}$.

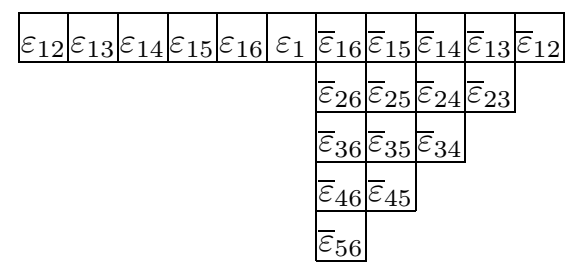

Figure 1: The (po)set of commutative roots for $\mathfrak{s o}_{13}$.

In such a diagram, the maximal element $\left(\theta=\bar{\varepsilon}_{12}\right)$ appears in the northeast corner and the smaller elements appear to the south and west. The edges correspond to the pairs of boxes having a common side. The direction of arrows is either ' $\rightarrow$ ' or ' $\uparrow$ '. This Ferrers diagram consists of the tail of length $n$ and the triangle 'of size $n-1$ '. The triangle itself represents an 
abelian ideal, and the structure of the set of ideals sitting inside this triangle is the same as for $\mathfrak{s p}_{2 n-2}$.

(i) Let us compute the number of abelian ideals $\mathfrak{a}$ with $k$ generators.

- By Theorem 6.2, the number of such ideals inside the triangle is equal to $\left(\begin{array}{c}n \\ 2 k\end{array}\right)$.

- Suppose that $\mathfrak{a}$ has the tail of length $m, m \geqslant 1$, i.e., $\mathfrak{a}$ has the generator $\varepsilon_{1}-\varepsilon_{n+2-m}$ in the upper row. Then the rest of this row (to the right) is also in the ideal, and the ideal is determined by its part lying in the triangle of size $n-2$, in the rows from 2 to $n-1$. The condition of being abelian means that $\mathfrak{a}$ cannot have elements from $m-1$ leftmost columns of the triangle. (Formally: if $\varepsilon_{1}-\varepsilon_{n+2-m} \in \mathfrak{a}$, then for all other roots $\varepsilon_{i}+\varepsilon_{j} \in \mathfrak{a}, 2 \leqslant i \leqslant j$, we must have $j \leqslant n+1-m$.) Hence as a degree of freedom for further constructing $\mathfrak{a}$ we have a triangle of size $n-1-m$, where an ideal with $k-1$ generators has to be chosen. The symplectic case shows that the number of such possibilities equals $\left(\begin{array}{c}n-m \\ 2 k-2\end{array}\right)$.

Thus, the total number of abelian ideals with $k$ generators equals

$$
\left(\begin{array}{c}
n \\
2 k
\end{array}\right)+\sum_{m \geqslant 1}\left(\begin{array}{c}
n-m \\
2 k-2
\end{array}\right)=\left(\begin{array}{c}
n \\
2 k
\end{array}\right)+\left(\begin{array}{c}
n \\
2 k-1
\end{array}\right)=\left(\begin{array}{c}
n+1 \\
2 k
\end{array}\right) .
$$

(ii) Let us compute the number of all abelian ideals $\mathfrak{a}$ with $k$ extensions. Here the argument is similar in the spirit, but more tedious.

- Suppose $\mathfrak{a}$ lies in the triangle. The difficulty here is that $\mathfrak{a}$ may have an extension that does not fit in the triangle (namely, if the length of the first row equals $n-1$ ). Therefore the symplectic formula does not immediately apply.

Let $p \leqslant n-1$ be the length of the first row of $\mathfrak{a}$. Then $\mathfrak{a}$ certainly have the extension in the first row. The rest of $\mathfrak{a}$ (in the second row and below) sits in the triangle of size $p-1$ and must have $k-1$ extensions. By the symplectic formula, the number of possibilities here is $\left(\begin{array}{c}p \\ 2 k-2\end{array}\right)$. Hence, the total number of possibilities for the ideals inside the triangle equals $\sum_{p \leqslant n-1}\left(\begin{array}{c}p \\ 2 k-2\end{array}\right)=\left(\begin{array}{c}n \\ 2 k-1\end{array}\right)$.

- Suppose an abelian ideal $\mathfrak{a}$ has the tail of length $m, m \geqslant 1$. Let $s$ be the length of the second row of $\mathfrak{a}$. Then, as explained in the proof of part (i), $s \leqslant n-m-1$. Here one has to distinguish two cases.

(1) If $s=n-m-1$, then $\mathfrak{a}$ has no extensions in the first two rows. Hence all $k$ extensions must occur in row no. 3 and below. This part of $\mathfrak{a}$ sits in the triangle of size $n-m-2$. Therefore one has $\left(\begin{array}{c}n-m-1 \\ 2 k\end{array}\right)$ possibilities for constructing an ideal. 
(2) If $s<n-m-1$, then $\mathfrak{a}$ has extensions in both the first and second row. Hence the lower part of $\mathfrak{a}$, in row no. 3 and below, must have $k-2$ extensions. Since this lower part sits inside the triangle of size $s-1$, one has $\left(\begin{array}{c}s \\ 2 k-4\end{array}\right)$ possibilities. Altogether, we obtain $\sum_{s \leqslant n-m-2}\left(\begin{array}{c}s \\ 2 k-4\end{array}\right)=\left(\begin{array}{c}n-m-1 \\ 2 k-3\end{array}\right)$ variants.

Thus, the total number of abelian ideals with $k$ extensions equals

$$
\begin{gathered}
\left(\begin{array}{c}
n \\
2 k-1
\end{array}\right)+\sum_{m \geqslant 1}\left(\begin{array}{c}
n-m-1 \\
2 k
\end{array}\right)+\sum_{m \geqslant 1}\left(\begin{array}{c}
n-m-1 \\
2 k-3
\end{array}\right) \\
=\left(\begin{array}{c}
n \\
2 k-1
\end{array}\right)+\left(\begin{array}{c}
n-1 \\
2 k+1
\end{array}\right)+\left(\begin{array}{c}
n-1 \\
2 k-2
\end{array}\right) .
\end{gathered}
$$

(iii) This follows from (i) and (ii) via a straightforward calculation.

Theorem 6.4. If $\mathfrak{g}=\mathfrak{s o}_{2 n}$, i.e., $\Delta$ is of type $\mathbf{D}_{n}$, then

(i) $\hat{\mathcal{K}}_{\mathfrak{A} \mathfrak{b}}(q)=\sum_{k \geqslant 0}\left(\left(\begin{array}{c}n+2 \\ 2 k\end{array}\right)-4\left(\begin{array}{c}n-1 \\ 2 k-2\end{array}\right)\right) q^{k}=\sum_{k \geqslant 0}\left(\left(\begin{array}{c}n \\ 2 k\end{array}\right)+\left(\begin{array}{c}n-1 \\ 2 k-1\end{array}\right)+\left(\begin{array}{c}n-2 \\ 2 k-1\end{array}\right)+\right.$ $\left.\left(\begin{array}{c}n-2 \\ 2 k-4\end{array}\right)\right) q^{k}$

(ii) $\check{\mathcal{K}}_{\mathfrak{A b}}(q)=\sum_{k \geqslant 0}\left(\left(\begin{array}{c}n \\ 2 k+1\end{array}\right)+\left(\begin{array}{c}n \\ 2 k-2\end{array}\right)\right) q^{k}$;

(iii) $\mathcal{D}_{\mathfrak{A} \mathfrak{b}}(q)=-\sum_{k \geqslant 0}\left(\left(\begin{array}{c}n-2 \\ 2 k+1\end{array}\right)+\left(\begin{array}{c}n-3 \\ 2 k\end{array}\right)\right) q^{k}$.

Proof. Here the set of commutative roots is

$$
\left\{\varepsilon_{i}+\varepsilon_{j} \mid 1 \leqslant i<j \leqslant n\right\} \cup\left\{\varepsilon_{1}-\varepsilon_{i} \mid 2 \leqslant i \leqslant n\right\} \cup\left\{\varepsilon_{i}-\varepsilon_{n} \mid 2 \leqslant i \leqslant n-1\right\} .
$$

This set is represented by a skew Ferrers diagram with row lengths $(2 n-$ $2, n-1, n-2, \ldots, 2)$. See the sample figure for $\Delta$ of type $\mathbf{D}_{6}$, where $\varepsilon_{i j}=$ $\varepsilon_{i}-\varepsilon_{j}$ and $\bar{\varepsilon}_{i j}=\varepsilon_{i}+\varepsilon_{j}$. The roots $\varepsilon_{1}-\varepsilon_{2}, \ldots, \varepsilon_{1}-\varepsilon_{n-1}$ form the tail of the Ferrers diagram for the set of commutative roots in type $\mathbf{D}_{n}$.

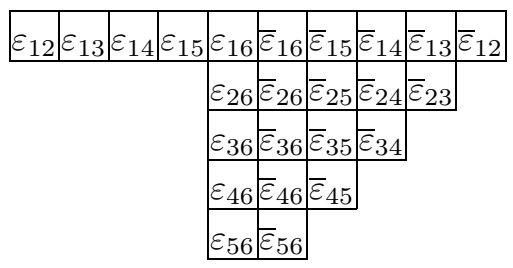

Figure 2: The (po)set of commutative roots for $\mathfrak{s o}_{12}$.

To a great extent, convention from the proof of Theorem 6.3 applies here. However, the notable distinction of this diagram from Figure 1 is that 
here the pairs of roots in the two central columns (i.e., $\varepsilon_{i n}, \bar{\varepsilon}_{i n}$ for $i<n$ ) are incomparable.

(i) Let us compute the number of abelian ideals $\mathfrak{a}$ with $k$ generators.

$\left(\nabla_{1}\right)$ Suppose that $\mathfrak{a}$ has the tail of length $m, m \geqslant 1$, i.e., $\mathfrak{a}$ has the generator $\varepsilon_{1}-\varepsilon_{n-m}$ in the upper row. Then the rest of this row (to the right) is also in the ideal, and the ideal is determined by its part lying in rows from 2 to $n-1$. The condition of being abelian means that $\mathfrak{a}$ cannot have elements from $m+2$ leftmost columns in this lower part. (Formally: if $\varepsilon_{1}-\varepsilon_{n-m} \in \mathfrak{a}$, then for all other roots $\varepsilon_{i}+\varepsilon_{j} \in \mathfrak{a}, 2 \leqslant i \leqslant j$, we must have $j \leqslant n-1-m$.) Hence as a degree of freedom for further constructing $\mathfrak{a}$ we obtain a triangle of size $n-3-m$, where an ideal with $k-1$ generators has to be chosen. The symplectic case shows that the number of possibilities equals $\left(\begin{array}{c}n-m-2 \\ 2 k-2\end{array}\right)$. Thus, in this case we have $\sum_{m \geqslant 1}\left(\begin{array}{c}n-m-2 \\ 2 k-2\end{array}\right)=\left(\begin{array}{c}n-2 \\ 2 k-1\end{array}\right)$ possibilities.

$\left(\mathcal{Q}_{2}\right)$ Suppose that an ideal $\mathfrak{a}$ has no tail, i.e., $\varepsilon_{1}-\varepsilon_{n-1} \notin \mathfrak{a}$. Consider all the relevant variants.

1. $\varepsilon_{1}-\varepsilon_{n}, \varepsilon_{1}+\varepsilon_{n} \in \mathfrak{a}$. These two roots are generators of $\mathfrak{a}$, so that we have to choose an ideal with $k-2$ generators in the triangle of size $n-3$. This yields $\left(\begin{array}{c}n-2 \\ 2 k-4\end{array}\right)$ possibilities.

2. $\varepsilon_{1}-\varepsilon_{n} \notin \mathfrak{a}$. Here we have to choose an ideal with $k$ generators in the triangle of size $n-1$. This yields $\left(\begin{array}{c}n \\ 2 k\end{array}\right)$ possibilities.

3. $\varepsilon_{1}+\varepsilon_{n} \notin \mathfrak{a}$. This part is the same as the previous one, and we obtain $\left(\begin{array}{c}n \\ 2 k\end{array}\right)$ possibilities.

4. In items (2) and (3), we have counted twice the ideals that contain neither $\varepsilon_{1}-\varepsilon_{n}$ nor $\varepsilon_{1}+\varepsilon_{n}$, i.e., the ideals with $k$ generators that fit in the triangle of size $n-2$. Therefore $\left(\begin{array}{c}n-1 \\ 2 k\end{array}\right)$ must be subtracted.

Thus, if $\mathfrak{a}$ has no tail, one obtains the sum $\left(\begin{array}{c}n-2 \\ 2 k-4\end{array}\right)+\left(\begin{array}{c}n \\ 2 k\end{array}\right)+\left(\begin{array}{c}n-1 \\ 2 k-1\end{array}\right)$.

Combining $\left(\nabla_{1}\right)$ and $\left(\nabla_{2}\right)$ yields the coefficients of $q^{k}$ presented as the sum of four summands. It is a good exercise to transform this sum into the second expression in the formulation.

(ii) Counting the ideals with $k$ extensions is even more tedious. Our approach yields 6 cases and 10 binomial coefficients, which sum luckily up to the two summands in the formulation. We only list all the possibilities for the Ferrers diagram and the corresponding number of ideals:

- the ideal has the tail (of length $\geqslant 1) \quad-\left(\begin{array}{c}n-3 \\ 2 k+1\end{array}\right)+\left(\begin{array}{c}n-3 \\ 2 k-2\end{array}\right)$

- the length of the first row is $\leqslant n-3 \quad-\left(\begin{array}{c}n-2 \\ 2 k-1\end{array}\right)$

- the length of the first row is $n-2 \quad-\left(\begin{array}{c}n-2 \\ 2 k-4\end{array}\right)$

- $\varepsilon_{1}-\varepsilon_{n} \notin \mathfrak{a}, \varepsilon_{1}+\varepsilon_{n} \in \mathfrak{a} \quad-\left(\begin{array}{c}n-2 \\ 2 k\end{array}\right)+\left(\begin{array}{c}n-2 \\ 2 k-3\end{array}\right)$ 
- $\varepsilon_{1}-\varepsilon_{n} \in \mathfrak{a}, \varepsilon_{1}+\varepsilon_{n} \notin \mathfrak{a}$

$$
\begin{aligned}
& -\left(\begin{array}{c}
n-2 \\
2 k
\end{array}\right)+\left(\begin{array}{c}
n-2 \\
2 k-3
\end{array}\right) \\
& -\quad\left(\begin{array}{c}
n-3 \\
2 k
\end{array}\right)+\left(\begin{array}{c}
n-3 \\
2 k-3
\end{array}\right)
\end{aligned}
$$

- $\varepsilon_{1} \pm \varepsilon_{n} \in \mathfrak{a}$

- the ideal has the tail (of length $\geqslant 1$ ) $-\left(\begin{array}{c}n-3 \\ 2 k+1\end{array}\right)+\left(\begin{array}{c}n-3 \\ 2 k-2\end{array}\right)$.

(iii) This follows from (i) and (ii) via a straightforward calculation.

Finally, we present the table with complete information about the covering and deviation polynomials for $\mathfrak{A} \mathfrak{b}(\mathfrak{g})$.

\begin{tabular}{|c|c|c|c|}
\hline $\mathfrak{g}$ & $\hat{\mathcal{K}}_{\mathfrak{A b}(\mathfrak{g})}$ & $\check{\mathcal{K}}_{\mathfrak{A b}(\mathfrak{g})}$ & $-\mathcal{D}_{\mathfrak{A} \mathfrak{b}(\mathfrak{g})}$ \\
\hline $\mathbf{A}_{n}$ & $\sum_{k \geqslant 0}\left(\begin{array}{c}n+1 \\
2 k\end{array}\right) q^{k}$ & $\sum_{k \geqslant 0}\left(\left(\begin{array}{c}n \\
2 k+1\end{array}\right)+\left(\begin{array}{c}n \\
2 k-2\end{array}\right)\right) q^{k}$ & $\sum_{k \geqslant 0}\left(\begin{array}{c}n-1 \\
2 k+1\end{array}\right) q^{k}$ \\
\hline $\mathbf{B}_{n}$ & $\sum_{k \geqslant 0}\left(\begin{array}{c}n+1 \\
2 k\end{array}\right) q^{k}$ & $\begin{array}{l}\sum_{k \geqslant 0}\left(\left(\begin{array}{c}n-1 \\
2 k+1\end{array}\right)+\left(\begin{array}{c}n \\
2 k-1\end{array}\right)\right. \\
\left.\quad+\left(\begin{array}{c}n-1 \\
2 k-2\end{array}\right)\right) q^{k}\end{array}$ & $\sum_{k \geqslant 0}\left(\begin{array}{c}n-2 \\
2 k+1\end{array}\right) q^{k}$ \\
\hline $\mathbf{C}_{n}$ & $\sum_{k \geqslant 0}\left(\begin{array}{c}n+1 \\
2 k\end{array}\right) q^{k}$ & $\sum_{k \geqslant 0}\left(\begin{array}{c}n+1 \\
2 k\end{array}\right) q^{k}$ & 0 \\
\hline $\mathbf{D}_{n}$ & $\sum_{k \geqslant 0}\left(\left(\begin{array}{c}n+2 \\
2 k\end{array}\right)-4\left(\begin{array}{c}n-1 \\
2 k-2\end{array}\right)\right) q^{k}$ & $\sum_{k \geqslant 0}\left(\left(\begin{array}{c}n \\
2 k+1\end{array}\right)+\left(\begin{array}{c}n \\
2 k-2\end{array}\right)\right) q^{k}$ & $\begin{array}{l}\sum_{k \geqslant 0}\left(\left(\begin{array}{c}n-2 \\
2 k+1\end{array}\right)\right. \\
\left.+\left(\begin{array}{c}n-3 \\
2 k\end{array}\right)\right) q^{k}\end{array}$ \\
\hline $\mathbf{E}_{6}$ & $1+25 q+27 q^{2}+11 q^{3}$ & $6+21 q+20 q^{2}+17 q^{3}$ & $5+6 q$ \\
\hline $\mathbf{E}_{7}$ & $1+34 q+60 q^{2}+30 q^{3}+3 q^{4}$ & $7+35 q+40 q^{2}+43 q^{3}+3 q^{4}$ & $6+13 q$ \\
\hline $\mathbf{E}_{8}$ & $1+44 q+118 q^{2}+76 q^{3}+17 q^{4}$ & $8+49 q+87 q^{2}+95 q^{3}+17 q^{4}$ & $7+19 q$ \\
\hline $\mathbf{F}_{4}$ & $1+10 q+5 q^{2}$ & $2+8 q+6 q^{2}$ & 1 \\
\hline $\mathbf{G}_{2}$ & $1+3 q$ & $1+3 q$ & 0 \\
\hline
\end{tabular}

Table 3: The covering and deviation polynomials for $\mathfrak{A} \mathfrak{b}(\mathfrak{g})$

Some observations related to Table 3:

1. For $\mathbf{A}_{2 n}$, we have $\operatorname{deg} \check{\mathcal{K}}_{\mathfrak{A} \mathfrak{b}}-\operatorname{deg} \hat{\mathcal{K}}_{\mathfrak{A} \mathfrak{b}}=1$. For all other cases the degrees are equal. Perhaps, the reason is that the Coxeter number is odd if and only if $\Delta$ is of type $\mathbf{A}_{2 n}$.

2. One may observe that there are several regularities in Table 3. For all classical series, both covering polynomials satisfy the recurrence relation

$$
\mathcal{K}_{\mathfrak{A} \mathfrak{b}\left(\mathbf{X}_{n}\right)}(q)=2 \mathcal{K}_{\mathfrak{A} \mathfrak{b}\left(\mathbf{X}_{n-1}\right)}(q)+(q-1) \mathcal{K}_{\mathfrak{A} \mathfrak{b}\left(\mathbf{X}_{n-2}\right)}(q),
$$

where $\mathbf{X} \in\{\mathbf{A}, \mathbf{B}, \mathbf{C}, \mathbf{D}\}$ and $\mathcal{K}$ is either $\hat{\mathcal{K}}$ or $\check{\mathcal{K}}$. Furthermore, the sequence $\mathbf{E}_{3}=\mathbf{A}_{2} \times \mathbf{A}_{1}, \mathbf{E}_{4}=\mathbf{A}_{4}, \mathbf{E}_{5}=\mathbf{D}_{5}, \mathbf{E}_{6}, \mathbf{E}_{7}, \mathbf{E}_{8}$ can be regarded as the 'exceptional' series, and for this series the same recurrence relation is satisfied for $\hat{\mathcal{K}}$ (but not for $\check{\mathcal{K}}$ ). Here one might wonder about the possible meaning of the polynomial $\hat{\mathcal{K}}$ corresponding to " $\mathbf{E}_{9}$ ". 
It also follows from (6.1) that $\mathcal{K}_{\mathfrak{A b}\left(\mathbf{X}_{n}\right)}(1)=2 \mathcal{K}_{\mathfrak{A} \mathfrak{b}\left(\mathbf{X}_{n-1}\right)}$ (1), which "explains" the equality $\# \mathfrak{A} \mathfrak{b}\left(\mathbf{X}_{n}\right)=2^{n}$.

3. The upper covering polynomials for $\mathbf{A}_{n}, \mathbf{B}_{n}, \mathbf{C}_{n}$ are the same. But the lower covering polynomial distinguishes these series. Furthermore, if the Dynkin diagram has no branching nodes, then $\hat{\mathcal{K}}_{\mathfrak{A} \mathfrak{b}}(\mathfrak{g})$ depends only on $n$. That is, the upper covering polynomial for $\mathbf{F}_{4}$ (resp. $\mathbf{G}_{2}$ ) is equal to that for $\mathbf{A}_{4}$ (resp. $\mathbf{A}_{2}$ ).

On the other hand, the lower covering polynomials are equal for $\mathbf{A}_{n}$ and $\mathbf{D}_{n}$, and the deviation polynomial for $\mathbf{B}_{n}$ is equal to that for $\mathbf{A}_{n-1}$.

It would be interesting to find an explanation of these coincidences.

Remark. For the non-reduced root system $\mathbf{B C}_{n}$, one can also consider combinatorial abelian ideals. However, these are exactly the same as in the symplectic case.

\section{Some questions and open problems}

$1^{o}$. Is there a combinatorial interpretation of the values $\hat{\mathcal{K}}_{\mathcal{P}}(-1)$ and $\check{\mathcal{K}}_{\mathcal{P}}(-1)$ ? Specifically, for the posets $\mathfrak{A} \mathfrak{d}, \mathfrak{A d}_{0}$, and $\mathfrak{A} \mathfrak{b}$, one might expect some characteristics of the corresponding root system.

$2^{o}$. Various examples considered in the paper show that in many cases the deviation polynomial of a poset has the nonzero coefficients of the same sign. It would be interesting to find a general pattern for this phenomenon. Of course, it is not always the case. For instance, if $\mathcal{D}_{\mathcal{P}_{1}}\left(\right.$ resp. $\mathcal{D}_{\mathcal{P}_{2}}$ ) has positive (resp. negative) coefficients, then Lemma 2.1(ii) shows that the deviation polynomial of $\mathcal{P}_{1} \times \mathcal{P}_{2}$ may have coefficients of both signs. It is not hard to produce a concrete example. However, I conjecture that the following is true:

Suppose that $\mathcal{P}=J^{*}(\mathcal{L})$, and let $\mathcal{P}(\leqslant m)$ be the subposet of upper ideals of $\mathcal{L}$ whose cardinality is at most $m$. Then $\mathcal{D}_{\mathcal{P}(\leqslant m)}$ has non-positive coefficients for all $m$.

In the special case of $J^{*}(\mathcal{L}) \backslash\{\hat{1}\}$, this is verified in Example 2.5.

$3^{o}$. Using Table 3 , one can compute the values $-\mathcal{D}_{\mathfrak{A} \mathfrak{b}}(1)$. In the serial cases, these values are quite simple:

$2^{n-2}$ for $\mathbf{A}_{n}, n \geqslant 2 ; 2^{n-3}$ for $\mathbf{B}_{n}, n \geqslant 3 ; 0$ for $\mathbf{C}_{n}, n \geqslant 1 ; 2^{n-3}+2^{n-4}$ for $\mathbf{D}_{n}, n \geqslant 4$.

Hopefully, there could be a uniform general description for them. One may notice that if the Dynkin diagram has no branching nodes, then this value equals $2^{m}$ with $m=\#\left(\Pi_{l}\right)-2$; or 0 , if $\#\left(\Pi_{l}\right)=1$. (The case of $\mathbf{G}_{2}$ and $\mathbf{F}_{4}$ is included here.) But I have no idea how to explain the values for series $\mathbf{D}$ and $\mathbf{E}$. 


\section{Acknowledgements}

This work was done during my visits to the Max-Planck-Institut für Mathematik (Bonn), and I thank the Institute for the hospitality and inspiring environment. I wish to thank F. Chapoton for sending me his unpublished notes and R. Stanley for drawing my attention to work of Dilworth [8].

\section{References}

[1] Athanasiadis, C. A. (2005). On a refinement of the generalized Catalan numbers for Weyl groups. Trans. AMS 357 179-196. MR2098091

[2] Bessis, D. (2003). The dual braid monoid, Ann. Sci. École Norm. Sup. Sér. IV 36 647-683. MR2032983

[3] Bourbaki, N. (1975). Groupes et algèbres de Lie, Chapitres 4, 5 et 6, Hermann, Paris. MR0453824

[4] Cellini, P. and Papi, P. (2000). ad-nilpotent ideals of a Borel subalgebra. J. Algebra 225 130-141. MR1743654

[5] Cellini, P. and Papi, P. (2002). ad-nilpotent ideals of a Borel subalgebra II. J. Algebra 258 112-121. MR1958899

[6] Chapoton, F. (2002). Data file on cluster graphs. Manuscript dated August 30.

[7] Chapoton, F. (2004). Enumerative properties of generalized associahedra. Sém. Lothar. Combin. 51 Art. B51b, 16 pp. (electronic). MR2080386

[8] Dilworth, R. P. (1954). Proof of a conjecture on finite modular lattices. Annals Math. 60 359-364. MR0063348

[9] Humphreys, J. E. (1992). Reflection Groups and Coxeter Groups. Cambridge Univ. Press. MR1066460

[10] Joseph, A. (1998). Orbital varieties of the minimal orbit. Ann. Sci. École Norm. Sup., Sér. IV, 31 17-45. MR1604290

[11] Kostant, B. (1998). The set of abelian ideals of a Borel subalgebra, Cartan decompositions, and discrete series representations. Intern. Math. Res. Notices (5) 225-252. MR1616913

[12] Krattenthaler, C. (2006). The F-triangle of the generalised cluster complex, in: Topics in Discrete Mathematics, Algorithms Combin., vol. 26, Springer, Berlin, pp. 93-126. MR2249265 
[13] Panyushev, D. (1999). On spherical nilpotent orbits and beyond, Ann. Inst. Fourier 49 1453-1476. MR1723823

[14] Panyushev, D. (2003). Abelian ideals of a Borel subalgebra and long positive roots. Intern. Math. Res. Notices (35) 1889-1913. MR1995141

[15] Panyushev, D. (2004). ad-nilpotent ideals of a Borel subalgebra: Generators and duality. J. Algebra 274 822-846. MR2043377

[16] Panyushev, D. (2006). The poset of positive roots and its relatives. $J$. Algebraic Combin. 23 79-101. MR2218851

[17] Panyushev, D. (2009). Properties of weight posets for weight multiplicity free representations. Moscow Math. J. 9(4) 867-883. MR2663994

[18] Panyushev, D. and Röhrle, G. (2001). Spherical orbits and abelian ideals. Adv. Math. 159 229-246. MR1825058

[19] Reiner, V. and Welker, V. (2005). On the Charney-Davis and NeggersStanley conjectures. J. Combin. Theory. Ser. A 109(2) 247-280. MR2121026

[20] Röhrle, G. (1998). On normal abelian subgroups of parabolic groups. Ann. Inst. Fourier 48 1455-1482. MR1662259

[21] Sommers, E. (2005). B-stable ideals in the nilradical of a Borel subalgebra. Canad. Math. Bull. 48 460-472. MR2154088

[22] Stanley, R. P. (1997). Enumerative Combinatorics, vol. 1. Cambridge Univ. Press. MR1442260

[23] Stembridge, J. R. (1986). Trapezoidal chains and antichains. Europ. J. Combin. 7 377-387. MR0868555

DMitri PANyUSheV

INDEPENDENT UNIVERSITY OF MOSCOW

Bol'shoi VlaseVSKII PER. 11

119002 Moscow

RUsSIA

Institute FOR INFORMATION TRANSMission PROBLEMS

B. Karetnyi PeR. 19

127994 Moscow

Russia

E-mail address: panyushev@iitp.ru

Received February 6, 2011 